\title{
In-silico Studies on Virulence Factors of Cryptococcus Species: Phylogenetic Analysis and B-cell Epitope Prediction
}

\author{
Kriti Kumari $^{1}{ }^{(\mathbb{D})}$, Gunjan Uttam ${ }^{2} \mathbb{( D}$, Karuna Singh ${ }^{2, *}$, Ankita Kumari $^{2}{ }^{(\mathbb{D}}$, Neha Nidhi Tirkey ${ }^{2}$ (D) \\ 1 Department of Bioinformatics, Mahila Mahavidyalaya, Banaras Hindu University, Varanasi-221005, India \\ 2 Animal Mycology Laboratory, Department of Zoology, Mahila Mahavidyalaya, Banaras Hindu University, Varanasi- \\ 221005, India \\ * Correspondence: karunazoobhu@gmail.com;
}

Scopus Author ID 57213994745

Reeived: 2.03.2021; Revised: 2.03.2021; Accepted: 4.03.2021; Published: 31.03.2021

\begin{abstract}
Virulence proteins ensure the survival of Cryptococcus in its host. The epitopes present in these virulence factors can modulate the host's immune system and contribute tocryptococcosis's pathobiology significantly. The amino acid sequences of virulence factors (glucuronoxylomannan (GXM), superoxide dismutase (SOD), mannoprotein (MP), urease, CAP binding protein, galactoxylomannan (GalXM), phospholipase-B, and laccase) of $C$. neoformans, $C$. $n$. grubii, and $C$. gattii were retrieved from NCBI. Analyses of the phylogenetic relationship between virulence factors were performed by using PhyML software and JMP 13.1 software. Further, ABCpred, BCPred, BcePred web servers were employed for the prediction of linear B-cell epitopes in amino acid sequences of said virulence factors. In all the three Cryptococcus species, laccase, CAP binding protein, and mannoprotein were highly conserved compared to GalXM, GXM, and SOD virulence factors. Superoxide dismutase (SOD) with the lowest gamma distribution value is considered to be highly adaptable. Further, the maximum number of B-cell epitopes was observed on the urease of C. n. grubii. In due course of time, $\mathrm{Cu}, \mathrm{Zn}$ Superoxide dismutase (SOD) might play the main role in Cryptococcus species' pathogenicity due to its highly variable nature. Additionally, urease could be used to design epitope-based anti-cryptococcal drugs. Nonetheless, the results of this in-silico study need wet lab validation.
\end{abstract}

Keywords: virulence factors; Cryptococcus; in-silico; phylogenetic analysis; B-cell epitope.

(C) 2021 by the authors. This article is an open-access article distributed under the terms and conditions of the Creative Commons Attribution (CC BY) license (https://creativecommons.org/licenses/by/4.0/).

\section{Introduction}

Amid the emergence and re-emergence of microbial infections, it has become important to re-evaluate their pathogenicity. Like other microbes, fungal pathogens have also been adding new dimensions in their pathogenic potential by gaining novel virulence factors and honing the existing ones. Cryptococcus is an opportunistic pathogen of humans and animals. It was considered a 'sleeping giant among fungal diseases [1], but now it has become an 'awakening giant' [2]. Cryptococcus can adapt to varying conditions. Regulation and expression of virulence factors facilitate its transition from the environment to mammalian niches [3]. The major virulence factors of Cryptococcus species are capsule, glucuronoxylomannan (GXM), mannoprotein (MP), galactoxylomannan (GalXM), laccase, superoxide dismutase (SOD), urease, CAP binding protein, and phospholipase-B. 
The mucinous capsule of Cryptococcus is primarily composed of polysaccharides having a backbone of $\alpha-1,3-\mathrm{D}$-mannopyranose units with single residues of $\beta$-Dglucuronopyranosyl and B-D-xylopyranosyl [4,5]. It is regarded as an important virulence factor and is anti-phagocytic [6]. GXM is the major component of the cryptococcal capsule, which plays a key role in organism's pathogenicity [7]. It is also a superlative target for host antibodies [8]. The capsule also contains transient components MP and GalXM, destined for cellular export [9].

Different enzymes and proteins contribute to the virulence of fungal pathogens. The high urease activity ensures the survival of Cryptococcus in avian guano by metabolizing uric acid, xanthines, and creatinine [10]. It helps the fungus to cross the blood-brain barrier, thereby contributing to the pathogenesis of Cryptococcus [11-13] crucially. Laccase is considered a major virulence factor of Cryptococcus species. It is responsible for melanin synthesis, which enhances fungal cells' resistance against attack by host effector mechanisms [14,15].

The antioxidant defense of Cryptococcus species largely relies on $\mathrm{Cu}, \mathrm{Zn}$ superoxide dismutase (SOD) [16,17]. SOD catalyzes the conversion of superoxide to hydrogen peroxide and oxygen water and facilitates the growth of Cryptococcus species within macrophages by protecting the host's superoxide. Its production increases at $37^{\circ} \mathrm{C}$, thus protecting the fungus from the oxidative damage generated by the host. $[18,19]$. Like other antioxidant enzymes, $\mathrm{Cu}$, $\mathrm{Zn}$ superoxide dismutase (SOD) is important for host predilection in Cryptococcus species.

Biologically active compounds formed by the cleaving action of phospholipase on phospholipids can alter the microenvironment and facilitate the survival of $C$. neoformans in the host. [20]. The extracellular phospholipase disrupts mammals' cell membranes and helps penetrate yeast cells into host tissues [21].

All epitopes of the pathogen are not able to elicit an immune response in the host. Insilico selection using bioinformatics tools can identify potentially immunoprotective epitopes [22-23]. ABCpred, BCPred, and BcePred web servers predict continuous B-cell epitopes based on secondary structure, hydrophilicity, exposed surface area, polarity, flexibility, and charge of amino acids [24-25], whereas DiscoTope, BEpro, and SEPPA use the 3-D structure of protein antigen as input [26]. The predicted epitopes can accelerate the formulation of epitopebased vaccine candidates [21]. Extracellular enzymes like laccase, urease, components of capsule viz. GXM, MP, GalXM, and chitosan of the cell wall are promising targets for antifungal drugs [11, 27-29].

The present study reports phylogenetic analysis and prediction of B cell epitopes of the virulence factors of $C$. n. neoformans, C. n. grubii, and C. gattii using a bioinformatics approach.

\section{Materials and Methods}

\subsection{Sequences retrieval.}

The amino acid sequences of CAP binding proteins, laccase, urease, SOD, phospholipase-B, GalXM, MP, GXM of C. n. neoformans, C. n. grubii, and C. gattii were retrieved from NCBI (National Center of Biotechnology Information, www.ncbi.nlm.nih.gov) in FASTA format. The orthologs were found by performing a BLAST search (https://blast.ncbi.nlm.nih.gov). 
2.2. Maximum likelihood test and hierarchical clustering of virulence proteins of Cryptococcus species.

Retrieved sequences of CAP binding proteins, laccase, urease, SOD, phospholipase-B, GalXM, MP, GXM of $C . n$. neoformans, $C$. n. grubii and $C$. gattii were aligned through MUSCLE software (https://www.ebi.ac.uk/Tools/msa/muscle/) [30] in clustal format. Further, these sequences were submitted for testing the likelihood using the PhyML web server (http://phylogeny.lirmm.fr/phylo_cgi/one_task.cgi?task_type=phyml) [31]. Protein datatype and WAG (substitution model) were used in PhyML with default settings for likelihood test.

$\begin{array}{lllll}\text { Further, clustering was done using software JMP } & \text { 13.1. }\end{array}$ (https://www.jmp.com/en_gb/download-jmp-free-trial.html). Clustering was done by the 'ward method'. Data of similar value were grouped to form a cluster.

\subsection{B-cell linear epitope prediction in virulence proteins of Cryptococcus species.}

Amino acid sequences of CAP binding proteins, laccase, urease, SOD, phospholipaseB, GalXM, MP, GXM of C. n. neoformans, $C$. $n$. grubii, and $C$. gattii. were submitted to ABCpred (http://crdd.osdd.net/raghava/abcpred/), BCPred (http://ailab.ist.psu.edu/bcpred/predict.html) and BcePred webservers (http://crdd.osdd.net/raghava/bcepred/) [32]. These web servers were used to predict B-cell epitopes and the antigenicity of protein sequences. In the ABCpred server, overlapping filters were used. The threshold and window length parameters chosen for prediction were 0.70 and 20 , respectively. In the Bcepred server default threshold for all physicochemical properties was selected, and overlapping filters were used. Specificity percentage and epitope length parameters used for prediction were 75 and 20, respectively.

\section{Results and Discussion}

Its virulence factors determine the pathogenicity of an organism. The gain or loss of virulence is an adaptive mechanism governed by natural selection. Virulence is expressed only in a susceptible host [33]. Cryptococcus species can infect and cause disease in humans and a variety of animals. It has been suggested that virulence in C.neoformans and C. gattii may originate from surviving in its primary niche, the soil or avian guano. The presence of virulence factors in non-virulent strains of Cryptococcus indicates selection pressure incurred by environmental predators [34]. Environmental factors also contributed to Cryptococcus pathogenicity [35]. The virulence factors inherent to Cryptococcus include glucuronoxylomannan (GXM), superoxide dismutase (SOD), mannoprotein (MP), urease, CAP binding protein, galactoxylomannan (GalXM), phospholipase-B, and laccase. Although all these factors are essential for virulence, they could not predict the pathogenicity of the strain.

\subsection{Sequence retrieval.}

The amino acid sequences of different virulence proteins of C. n. neoformans, $C . n$. grubii, and C. gattii were retrieved from NCBI in FASTA format (Table 1). 
Table 1 List of virulence factors of Cryptococcus species retrieved from NCBI.

\begin{tabular}{|c|c|c|c|}
\hline $\begin{array}{l}\text { S.n } \\
\text { o. }\end{array}$ & $\begin{array}{l}\text { Virulence } \\
\text { factors }\end{array}$ & $\begin{array}{l}\text { Accession } \\
\text { No. }\end{array}$ & Sequence in FASTA format \\
\hline \multirow[t]{5}{*}{1.} & \multirow[t]{5}{*}{ Laccase } & ACB05902.1 & $\begin{array}{l}\text { >ACB05902.1 laccase } 1 \text { [Cryptococcus gattii VGI] } \\
\text { MRGIVNFFFLSCSLILLVSSENTGKLPTATIDPSVFALSNDFEVSDVPTTREYT } \\
\text { FDITKALASPDGYEREVYTVNNMFPGPVIEANTGDTIIVHVNNHLDKGQSIH } \\
\text { WHGMRQKDTPYMDGVPGITQCPIPPGGSYTYNFTISDQSGTYWWHSHYSN } \\
\text { AMADGLWGPLIVHSVHEPIQRGRDYDEDRIVFVSDWLHDDSEIIIAALATPA } \\
\text { GYRGSPAPPQGDSILINGRGQTNCTATNSSSCYYPAPPEIHVPVNSRVRLRFI } \\
\text { SATAHPMYRISLDNHPLEIVETDGTAVYGPTVHEMSIAPGERYSAIINTSEGK } \\
\text { EGDAFWLRTSVALGCMFGGVPQVGLAVVRYTGNEMTTTAEPQSYAWSDL } \\
\text { ANATALCAGLDQTYTLSPRERESCRVSRASSQSHIFNSQRGAFVNYLGNTF } \\
\text { QGYGFNNISYQNKIFDPILSMVQRGDPYESTLVASTTFPDMGAGAIINNLDG } \\
\text { PIDHPYHLHGNEFQVIGRGTGALSLDNLTNIEFSLDNPVRKDTLWIQGGSWA } \\
\text { ALSITTDNPGVWALHCHIGWHLTEGKLAVVVVQPNAVGQIACPESWTNLC } \\
\text { ANTDPNAFGPARRSPSPSIQSSKTSAFQRLREVKGNVKRRGAREV }\end{array}$ \\
\hline & & BAG50325.1 & $\begin{array}{l}\text { >BAG50325.1 diphenol oxidase [Cryptococcus neoformans var. grubii] } \\
\text { MRGVVKLFFLSCSFVSLVSSEETGKSPTANYDHYMPKATATIDPSVFALSND } \\
\text { FEITDVPTTREYTFDITKALASPDGYEREVYVVNNMFPGVIEANTGDTIIVH } \\
\text { VNNHLEEGQSIHWHGLRQLGTAFMDGVPGITQCPIPPGSSFTYQFTVSHQSG } \\
\text { TFWWHSHYSNSMADGIWGPLIIHSPNEPLQRGRDYDEDRIVFITDWVHDNS } \\
\text { EVVIAALATPEGYKGSPAPPQGDAILINGRGQTNCTATGSSSCTYPPPPEIHV } \\
\text { PVNCRVRLRFISATAHPMYRITIDNHPLEVVETDGTAVYGPTVHEISIAPGER } \\
\text { YSAIINTSEGKEGDAFWLRTSVALGCMFGGIDQVGLAVVRTGNGMVSTE } \\
\text { EPQTTAWSDLAGATTPCAGLDQTYTLSPRESFSAPREFSQSHVFNSQRGAFV } \\
\text { NVYGNTFQGYGFNNISYQNQIFNPLLSIVQRGGSCESTLVASTTFPDLGSGNI } \\
\text { IINNLDGVIDHPYHLHGNEFQVIGRGTGALSLDNLTNIDFNLDNPVRKDTLW } \\
\text { IQGGSWVVLRITTDNPGVWALHCHIGWHLTEGKLAVVVIQPGAIGHMEGP } \\
\text { ESWTNLCANTDPNAFGPARRSPSPSIQSSKTSTFQYLREVKGKVVKRRGAR } \\
\text { EA }\end{array}$ \\
\hline & & BAG50331.1 & $\begin{array}{l}\text { >BAG50331.1 diphenol oxidase [Cryptococcusneoformans var. neoformans] } \\
\text { MRGLAKLFFLSCSFVSLVSSEKTDESPTAVSDNYMPKATATIDPSVFALSND } \\
\text { FEITDVPTTREYTFDIAKAFASPDGYEREVYVVNNMFPGPIEANTGDTIIVH } \\
\text { VNNHLDEGQSLHWHGLRQLGTAFMDGVPGITQCPIPPGGSFTYNFTVSHQS } \\
\text { GTFWWHSHYSNSMADGIWGPLIVHSTNEPLQRGRDYDEDRIVFITDWMHD } \\
\text { NSEIIIAALATPEGYKGNIAPPQGDAILINGRGQTNCTATGSSSCFYPPPPEIQ } \\
\text { VPVNCRVRLRFISATAHPMYRISIDNHPMEVVEADGTAVYGPTVHEISVAPG } \\
\text { ERYSAIINTNEGKEGDAFWLRTSVALSCMFGAVSQEGLAVVRYTGNGMVS } \\
\text { TEEPQTSAWSDLAGVTVPCTGLDQTYTLSPRDSLSAPREPLQSHFFNSERGA } \\
\text { FVNVLGNTFQGYGFNNISYQNQIFNPLLSIVQRGGSCENTLVSSRTFPDFGPG } \\
\text { NIIINNLDTVIDHPYHLHGNEFQVIGRGTGALSIDNLTNIDFTLDNPVRKDTL } \\
\text { WIQGGSWAVLRITADNPGVWALHCHIGWHLTEGKLAVIVVQPSAIGHMES } \\
\text { PESWTNLCANTDPNAFGPARRSSSPSIQSSKTSSFQYLREVKGKVVKRRGAR } \\
\text { EA }\end{array}$ \\
\hline & & KIR41803.1 & $\begin{array}{l}\text { >KIR41803.1 laccase [Cryptococcus gattii VGII Ram5] } \\
\text { MRGIVNFFFLSCSLILLVSSENTGKLPTATIDPSVFALSNDFEVSDVPTTREYT } \\
\text { FDITKALASPDGYEREVYTVNNMFPGPVIEANTGDTIIVHVNNHLDKGQSIH } \\
\text { WHGMRQKDTPYMDGVPGITQCPIPPGGSYTYNFTISDQSGTYWWHSHYSN } \\
\text { AMADGLWGPLIVHSVHEPIQRGRDYDEDRIVFVSDWLHDDSEIIIAALATPA } \\
\text { GYRGSPAPPQGDSILINGRGQTNCTATNSSSCYYPAPPEIHVPVNSRVRLRFI } \\
\text { SATAHPMYRISLDNHPLEIVETDGTAVYGPTVHEMSIAPGERYSAIINTSEGK } \\
\text { EGDAFWLRTSVALGCMFGGVPQVGLAVVRYGNEMTTTAEPQSYAWSDL } \\
\text { ANATALCAGLDQTYTLSPRERESCRVSRASSQSHIFNSQRGAFVNYLGNTF } \\
\text { QGYGFNNISYQNKIFDPILSMVQRGDPYESTLVASTTFPDMGPGAIIINNLDG } \\
\text { PIDHPYHLHGNEFQVIGRGTGALSLDNLTNIEFSLDNPVRKDTLWIQGGSWA } \\
\text { ALGITTDNPGVWALHCHIGWHLTEGKLAVVVVQPNAVGQIACPESWTNLC } \\
\text { ANTDPNAFGPARRSPSPSIQSSKTSAFQRLREVKGNVKRRGAREV }\end{array}$ \\
\hline & & BAG50349.1 & $\begin{array}{l}\text { >BAG50349.1 diphenol oxidase [Cryptococcus neoformans] } \\
\text { MRGIVNFFFLSCSLILLVSSENTGKLPTATIDPSVFALSNDFEVSDVPTTREYT } \\
\text { FDITKALASPDGYEREVYTVNNMFPGPVIEANTGDTIIVHVNNHLDKGQSIH } \\
\text { WHGMRQKDTPYMDGVPGITQCPIPPGGSYTYNFTISDQSGTYWWHSHYSN } \\
\text { AMADGLWGPLIVHSVHEPIQRGRDYDEDRIVFVSDWLHDDSEIIIAALATPA } \\
\text { GYRGSPAPPQGDSILINGRGQTNCTATNSSSCYYPAPPEIHVPVNSRVRLRFI } \\
\text { SATAHPMYRISLDNHPLEIVETDGTAVYGPTVHEMSIAPGERYSAIINTSEGK } \\
\text { EGDAFWLRTSVALGCMFGGVPQVGLAVVRYGNEMTTAEPQSYAWSDLA } \\
\text { NATALCAGLDQTYTLSPRERESCRVSRASSQSHIFNSQRGAFVNYLGNTFQ } \\
\text { GYGFNNISYQNKIFDPILSMVQRGDPYESTLVASTTFPDMGAGAIIINNLDGP } \\
\text { IDHPYHLHGNEFQVIGRGTGALSLDNLTNIEFSLDNPVRKDTLWIQGGSWA } \\
\text { ALSITTDNPGVWALHCHIGWHLTEGKLAVVVVQPNAVGQIACPESWTNLC } \\
\text { ANTDPNSFGPARRSPSPSIQSSKTSAFQRLREVKGNVKRRGAREV }\end{array}$ \\
\hline
\end{tabular}




\begin{tabular}{|c|c|c|c|}
\hline \multirow[t]{2}{*}{$\begin{array}{l}\text { S.n } \\
\text { o. }\end{array}$} & $\begin{array}{l}\text { Virulence } \\
\text { factors }\end{array}$ & $\begin{array}{l}\text { Accession } \\
\text { No. }\end{array}$ & Sequence in FASTA format \\
\hline & & KIR33454.1 & $\begin{array}{l}\text { >KIR33454.1 laccase [Cryptococcus gattii VGII MMRL2647] } \\
\text { MRGIVNFFFLSCSLILLVSSENTGKLPTATIDPSVFALSNDFEVSDVPTTREYT } \\
\text { FDITKALASPDGYEREVYTVNNMFPGPVIEANTGDTIIVHVNNHLDKGQSIH } \\
\text { WHGMRQKDTPYMDGVPGITQCPIPPGGSYTYNFTISDQSGTYWWHSHYSN } \\
\text { AMADGLWGPLIVHSVHEPIQRGRDYDEDRIVFVSDWLHDDSEIIIAALATPA } \\
\text { GYRGSPAPPQGDSILINGRGQTNCTATNSSSCYYPAPPEIHVPVNSRVRLRFI } \\
\text { SATAHPMYRISLDNHPLEIVETDGTAVYGPTVHEMSIAPGERYSAIINTSEGK } \\
\text { EGDAFWLRTSVALGCMFGGVPQVGLAVVRYTGNEMTTTAEPQSYAWSDL } \\
\text { ANVTALCAGLDQTYTLSPRERESCRVSRASSQSHIFNSQRGAFVNYLGNTF } \\
\text { QGYGFNNISYQNKIFDPILSMVQRGDPYESTLVASTTFPDMGAGAIINNLDG } \\
\text { PIDHPYHLHGNEFQVIGRGTGALSLDNLTNIEFSLDNPVRKDTLWIQGGSWA } \\
\text { ALSITTDNPGVWALHCHIGWHLTEGKLAVVVVQPNAVGQIACPESWTNLC } \\
\text { ANTDPNAFGPARRSPSPSIQSSKTSAFQRLREVKGNVKRRGAREV }\end{array}$ \\
\hline \multirow[t]{3}{*}{2.} & \multirow[t]{3}{*}{ Urease } & AAC62257.1 & $\begin{array}{l}\text { >AAC62257.1 urease [Cryptococcus neoformans var. grubii] } \\
\text { MHLLPRETDKLILTTLGTLAQRRLARGLILNRAETIALISSQLQEFVRDGRHS } \\
\text { VAELMDLGKKMLGRRHVRKGVPESIHTIQVEGTFPDGVFLVTVDDPISSDD } \\
\text { GDLNNAFYGSFLPIPSADVFPAAPEPADTLLGALICRKETVKINASRRRFRLE } \\
\text { VKNAGDRPVQVGSHYHFLETNPALIFDRLLSYGYHLDIPAGTAVRFEPGEK } \\
\text { KTVTMVEFGGKKIFHGGSGLGNGSFDENLRETKVKEMVEKVGFGHKEQEK } \\
\text { IEEGPVTEMNREVYASMFGPTTGDKIKLADMDLWIEVEKDYTVYGDECKF } \\
\text { GGGKVIRDGGGQASGRHDHEVLDLVITNALIVDWTGIYKADIGVKNGIIVGI } \\
\text { GKAGNPDMMDGVTDGMIVGSSTEVISGEKLITTAGRLDVHVHYISPQLMTE } \\
\text { ALASGITTVIGGGTGPADGSNATTCTSSSFYMQNMIKATDTIPLNFGFTGKG } \\
\text { SDSGTNAMRDIIEAGACGLKVHEDWGATAEVIDRALSMADEYDVQINLHS } \\
\text { DTLNESGYVESTLAAIKGRTIHSYHTEGAGGGHAPDIIVVCEYENVLPSSTN } \\
\text { PTRPYAVNTLDEHLDMLMICHGLDKSIPEDIAFADSRIRSETVAAEDVLQDT } \\
\text { GAISMISSDCQAMGRIGEVVTRTWRTAAKMKQFRGPLEGDEPTRDNNRVK } \\
\text { RYVAKYTINPAITHGMSHLIGQVAVGCLADLVLLGESFGARPEMILKGGV } \\
\text { IAWAAVGDANASIPTVQPVLGRPMWALSLRPLHSIQLFVSQASLDKDLVKR } \\
\text { YRLRKRAEAVKNCRSIGKKDMKWNDTMPKMTVDPETYDVRADGVLCDV } \\
\text { PPADKLPLTRRYFVY }\end{array}$ \\
\hline & & $\begin{array}{l}\text { XP_01205364 } \\
4.1\end{array}$ & $\begin{array}{l}\text { >XP_012053644.1 urease [Cryptococcusneoformans var. grubii H99] } \\
\text { MSTDGTGWETKPHKGTQLPHPLRPVDSLLLFFIFCFPFPPHHTKNIMHLLP } \\
\text { RETDKLILTTLGTLAQRRLARGLILNRAETIALISSQLQEFVRDGRHSVAELM } \\
\text { DLGKKMLGRRHVKGVPESIHTIQVEGTFPDGVFLVTVDDPISSDDGDLNN } \\
\text { AFYGSFLPIPSADVFPAAPEPADTLLGALICRKETVKINASRRRFRLEVKNAG } \\
\text { DRPVQVGSHYHFLETNPALIFDRLLSYGYHLDIPAGTAVRFEPGEKKTVTM } \\
\text { VEFGGKKIFHGGSGLGNGSFDENLRETKVKEMVEKGGFGHKEQEKIEEGPV } \\
\text { TEMNREVYASMFGPTTGDKIKLADMDLWIEVEKDYTVYGDECKFGGGKVI } \\
\text { RDGGGQASGRHDHEVLDLVITNALIVDWTGIYKADIGVKNGIIVGIGKAGN } \\
\text { PDMMDGVTDGMIVGSSTEVISGEKLITTAGALDVHVHYISPQLMTEALASGI } \\
\text { TTVIGGGTGPADGSNATTCTSSSFYMQNMIKATDTIPLNFGFTGKGSDSGTN } \\
\text { AMRDIIEAGACGLKVHEDWGATPEVIDRALSMADEYDVQINLHSDTLNESG } \\
\text { YVESTLAAIKGRTIHSYHTEGAGGGHAPDIIVVCEYENVLPSSTNPTRPYAV } \\
\text { NTLDEHLDMLMICHGLDKSIPEDIAFADSRIRSETVAAEDVLQDTGAISMISS } \\
\text { DCQAMGRIGEVVTRTWRTAAKMKQFRGPLEGDEPTRDNNRVKRYVAKYT } \\
\text { INPAITHGMSHLIGQVAVGCLADLVFWTAESFGARPEMILKGGVIAWAAVG } \\
\text { DANASIPTVQPVLGRPMWGSQPEAAALNSIVWVSQASLDKDLVKRFNIKKR } \\
\text { AEAVKNCRSIGKKDMKWNDTMPKMTVDPETYDVRADGVLCDVPPADKLP } \\
\text { LTRRYFVY }\end{array}$ \\
\hline & & XP_572365.1 & $\begin{array}{l}\text { >XP_572365.1 urease [Cryptococcus neoformans var. neoformans JEC21] } \\
\text { MHLLPRETDKLIVTTLGTLAQRRARGLILNRAETIALISSQLQEFIRDGRHS } \\
\text { VAELMDLGKKMLGRRHVRKGVPESIHTIQVEGTFPDGVFLVTVDDPISSDD } \\
\text { GDLNNAFYGSFLPIPSADVFPAAPEPADTLLGALICRKEPIKINASRRRFLE } \\
\text { VKNAGDRPIQVGSHYHFLETNPALIFDRLLSYGYHLDIPAGTAVRFEPGEKK } \\
\text { TVTMVEFGGKKIFHGGSGLASGSFDENLRETKVKEMVEKGGFGHKDQEKV } \\
\text { EEGPTTEMNREVYASMFGPTTGDKIKLADMDLWIEVEKDYTVYGEECKFG } \\
\text { GGKVLRDGGGQASGRHEHEVLDLVITNALIVDWNGIYKADIGVKNGIIVGI } \\
\text { GKAGNPDMMDGVTDGMIVGSSTEVIAGEKLIITAGALDVHVHYICPQLMTE } \\
\text { ALASGITTVVGGGTGPADGSNATTCTSSSFYMQNMIKATDTVPLNFGFTGK } \\
\text { GNDSGTNALRDVIEAGACGLKVHEDWGATPEVIDRALSIADEYDVQVNLH } \\
\text { SDTLNESGYVESTLAAIKGRTIHSYHTEGAGGGHAPDIIVVCEYENVLPSST } \\
\text { NPTRPYAVNTLDEHLDMLMVCHHLDKSIPEDIAFADSRIRSETVAAEDVLQ } \\
\text { DTGAISMISSDCQAMGRIGEVITRTWRTAAKMKQFRGPLEGDEPTRDNNRV } \\
\text { KRYVAKYTINPAITHGMSHLIGQVAVGCLADLVFWTAESFGARPEMILKGG } \\
\text { VIAWAAMGDANASIPTVQPVIGRPMWGSQPEAAALNSIVWVSQASLDKDL } \\
\text { VKRFNIKKRAEAVKNCRAIGKKDMKWNDSMPKMTVDPETYDVHADGVL } \\
\text { CDVPPADKLPLTKRYFVY }\end{array}$ \\
\hline
\end{tabular}




\begin{tabular}{|c|c|c|c|}
\hline \multirow[t]{4}{*}{$\begin{array}{l}\text { S.n } \\
\text { o. } \\
\end{array}$} & $\begin{array}{l}\text { Virulence } \\
\text { factors }\end{array}$ & $\begin{array}{l}\text { Accession } \\
\text { No. }\end{array}$ & Sequence in FASTA format \\
\hline & & KGB78493.1 & $\begin{array}{l}\text { >KGB78493.1 urease [Cryptococcus gattii VGII R265] } \\
\text { MHLLPRETDKLILTTLGTLAQRRLARGLILNRAETIALISSQLQEFIRDGRHS } \\
\text { VAELMDMGKKMLGRRHVRKGVPESIHSIQVEGTFPDGVFLVTVDDPISSDD } \\
\text { GDLNNAFYGSFLPIPSADVFPAAPEPKDTLLGALICREEPIKINVSRRRFRLEV } \\
\text { KNAGDRPIQVGSHYHFLETNPALVFDRLLSYGYHLDIPAGTAVRFEPGEKK } \\
\text { TVTMVEFGGKKIFHGGSGLGSGPFNENLRETTIKAMVEKGGFSHKEQEKVE } \\
\text { EGPVTEMNREVYASMFGPTTGDKIKLADMDLWIEIEKDYTVYGEECKFGG } \\
\text { GKVLRDGGGQASGRYDHEVLDLVITNALIVDWNGIYKADIGVKNGIIVGIG } \\
\text { KAGNPDMMDGVTDGMIVGSNSEVIAGEKLIVTAGALDVHVHYICPQLMTE } \\
\text { ALASGITTVVGGGTGPADGSNATTCTSSPFYMQNMIKATDTMPLNFGFTGK } \\
\text { GNDSGTNSLRDIIEAGACGLKVHEDWGATPEVIDRALTIADEYDVQVNLHS } \\
\text { DTLNESGYVESTLAAIKGRTIHSYHTEGAGGGHAPDIIVVCEHENVLPSSTN } \\
\text { PTRPYAVNTLDEHLDMLMVCHHLDKSIPEDIAFADSRIRSETVAAEDVLQD } \\
\text { TGAISMISSDSQAMGRIGEVITRTWRTAAKMKQYRGPLEGDEPTRDNNRVK } \\
\text { RYVAKYTINPAITHGMSHLIGHVAVGCLADLVFWTAESFGARPEMVLKGG } \\
\text { VIAWAAIGEANAAIPTVQPVIGRPMWGAQPAAAALNSIVWVSQASLDKDL } \\
\text { VKRFDIKKRAEAVKNCRAIGKKDMKWNDTMPKMTVDPETYDVRADGVL } \\
\text { CDVPPADKLPLTKRYFVY }\end{array}$ \\
\hline & & KIR84016.1 & $\begin{array}{l}\text { >KIR84016.1 urease [Cryptococcus gattii VGIV IND107] } \\
\text { MHLLPRETDKLILTTLGTLAQRRLARGLILNRAETIALISSQLQEFIRDGRHS } \\
\text { VAELMDMGKKMLGRRHVRKGVPESIHSIQVEGTFPDGVFLVTVDDPISSDD } \\
\text { GDLNNAFYGSFLPIPSADVFPAAPEPKDTLLGALICRKEPIKINVSRRRFRLE } \\
\text { VKNAGDRPIQVGSHYHFLETNPALIFDRLLSYGYHLDIPAGTAVRFEPGEKK } \\
\text { TVTMVEFGGKKIFHGGSGLGSGPFNENLRDTKIKEMVEKGGFSHKEQEKVE } \\
\text { EGPVTEMNREVYASMFGPTTGDKIKLADMDLWIEIEKDYTVYGEECKFGG } \\
\text { GKVLRDGGGQASGRYDHEVLDLVITNALIVDWNGIYKADIGVKNGIIVGIG } \\
\text { KAGNPDMMDGVTDGMIVGSNSEVIAGEKLIVTAGALDVHVHYICPQLMTE } \\
\text { ALASGITTVVGGGTGPADGSNATTCTSSPFYMQNMIKATDTMPLNFGFTGK } \\
\text { GNDSGTNSLRDIIEAGACGLKVHEDWGATPEVIDRALTIADEYDVQVNLHS } \\
\text { DTLNESGYVESTLAAIKGRTIHSYHTEGAGGGHAPDIIVVCEHENVLPSSTN } \\
\text { PTRPYAVNTLDEHLDMLMVCHHLDKSIPEDIAFADSRIRSETVAAEDVLQD } \\
\text { TGAISMISSDSQAMGRIGEVITRTWRTAAKMKQYRGPLEGDEPTRDNNRVK } \\
\text { RYVAKYTINPAITHGMSHLIGHVAVGCLADLVFWTAESFGARPEMVLKGG } \\
\text { VIAWAAIGEANAAIPTVQPVIGRPMWGAQPAAAALNSIVWVSQASLDKDL } \\
\text { VKRFDIKKRAEAVKNCRAIGKKDMKWNDTMPKMTVDPETYDVRADGVL } \\
\text { CDVPPADKLPLTKRYFVY }\end{array}$ \\
\hline & & $\begin{array}{l}\text { XP_00319708 } \\
0.1\end{array}$ & $\begin{array}{l}\text { >XP_003197080.1 Urease (Urea amidohydrolase), putative [Cryptococcus gattii } \\
\text { WM276] } \\
\text { MHLLPRETDKLILTTLGTLAQRRLARGLILNRAETIALISSQLQEFIRDGRHS } \\
\text { VAELMDMGKKMLGRRHVRKGVPESIHSIQVEGTFPDGVFLVTVDDPISSDD } \\
\text { GDLNNALYGSFLPIPSADVFPAAPEPTDTLLGALICRKEPIKINVSRRRFRLEV } \\
\text { KNAGDRPIQVGSHYHFLETNPALIFDRLLSYGYHLDIPAGTAVRFEPGEKKT } \\
\text { VTMVEFGGKKIFHGGSGLGSGPFNENLRNTKIKEMVEKGGFSHKEQEKIEE } \\
\text { GPVTEMNREVYASMFGPTTGDKIKLADMDLWIEIEKDYTVYGEECKFGGG } \\
\text { KVLRDGGGQASGRYDHEVLDLVITNALIVDWNGIYKADIGVKNGIIVGIGK } \\
\text { AGNPDMMDGVTDGMIVGSNTEVIAGEKLIVTAGALDVHVHYICPQLMTEA } \\
\text { LASGITTVVGGGTGPADGSNATTCTSSPFYMQNMIKATDTMPLNFGFTGKG } \\
\text { NDSGTNSLRDIIEAGACGLKVHEDWGATPEVIDRALTIADEYDVQVNLHSD } \\
\text { TLNESGYVESTLAAIKGRTIHSYHTEGAGGGHAPDIIVVCEHENVLPSTNPT } \\
\text { RPYAVNTLDEHLDMLMVCHHLDKSIPEDIAFADSRIRSETVAAEDVLQDTG } \\
\text { AISMISSDSQAMGRIGEVITRTWRTAAKMKQYRGPLEGDEPTRDNNRVKRY } \\
\text { VAKYTINPAITHGMSHLIGHVAVGCLADLVFWTAESFGARPEMVLKGGVIA } \\
\text { WAAIGEANAAIPTVQPIIGRPMWGAQPAAAALNSIVWVSQASLDKDLVKRF } \\
\text { DIKKRAEAVKNCRAIGKKDMKWNDTMPKMTVDPETYDVRADGVLCDVPP } \\
\text { ADKLPLTKRYFVY }\end{array}$ \\
\hline \multirow[t]{2}{*}{3.} & \multirow[t]{2}{*}{ SOD } & XP_570285.1 & $\begin{array}{l}\text { >XP_570285.1 copper-zinc superoxide dismutase [Cryptococcus neoformans var. } \\
\text { neoformans JEC21] } \\
\text { MVKAVAVLKGDSHVYGTITFTQDSEGAPVCVSGEIKNLDADAKRGFHVHE } \\
\text { FGDNTNGCTSAGPHYNPFHKNHGGPTAAERHVGDLGNVQTNGCGVAMVD } \\
\text { ISDKVISLFGPHSIIGRSMVVHAGTDDLGKGGNEESLKTGNAGARLACGVIG } \\
\text { IAA }\end{array}$ \\
\hline & & KIR50011.1 & $\begin{array}{l}\text { >KIR50011.1 superoxide dismutase [Cu-Zn] [Cryptococcus gattii CA1280] } \\
\text { MLAVAVLKGDSPVTGVITFTQEKEGAPVTVSGDIKNLDANAERGFHVHEFG } \\
\text { DNTNGCTSAGPHFNPHGKNHGAPSDSERHVGDLGNVKTDSNGVASVNISD } \\
\text { KSLSLFGPYSIIGRTIVVHAGTDDFGKGGNAESLKTGNAGARAACGVIGISS }\end{array}$ \\
\hline
\end{tabular}




\begin{tabular}{|c|c|c|c|}
\hline \multirow[t]{5}{*}{$\begin{array}{l}\text { S.n } \\
\text { o. }\end{array}$} & $\begin{array}{l}\text { Virulence } \\
\text { factors }\end{array}$ & $\begin{array}{l}\text { Accession } \\
\text { No. }\end{array}$ & Sequence in FASTA format \\
\hline & & KGB74761.1 & $\begin{array}{l}\text { >KGB74761.1 superoxide dismutase [Cu-Zn] [Cryptococcus gattii VGII R265] } \\
\text { MPAVAVLKGDSPVTGVITFTQEKEGAPVTVSGDIKNLDANAERGFHVHEFG } \\
\text { DNTNGCTSAGPHFNPHGKNHGAPSDSERHVGDLGNVKTDGNGVASVNISD } \\
\text { KSLSLFGPYSIIGRTIVVHAGTDDFGKGGNPESLKTGNAGARAACGVIGISN }\end{array}$ \\
\hline & & AAK01665.1 & $\begin{array}{l}\text { >AAK01665.1 Cu/Zn superoxide dismutase [Cryptococcus neoformans var. grubii] } \\
\text { MVKAVVVLKGESYAHGIVCFTQESENAPVCITGEIKDMDADAKRGMHVHE } \\
\text { FGDNTNGCTSAAPHYNPFKKHHGAPTDSERHVGDLGNIQTNSCGAAQLDF } \\
\text { SDKIISLYGPHSIIGGSFVVHASTDDLGKGGNEESLKTGNAGARLACGVIGIS } \\
\text { TCQCYHSKLIVFAAVFLPKRTVTTYSWLNK }\end{array}$ \\
\hline & & $\begin{array}{l}\text { XP_00319350 } \\
4.1\end{array}$ & $\begin{array}{l}\text { >XP_003193504.1 copper zinc superoxide dismutase [Cryptococcus gattii WM276] } \\
\text { MRAVAVLKGDSPVTGVITFTQEKEGAPVTVSGDIKNLDANAERGFHVHEF } \\
\text { GDNTNGCTSAGPHFNPHGKNHGAPSDSERHVGDLGNVKTDGNGVASVNIS } \\
\text { DKSLSLFGPYSIIGRTIVVHAGTDDFGKGGNAESLKTGNAGARAACGVIGIS } \\
\mathrm{N}\end{array}$ \\
\hline & & KIR29693.1 & $\begin{array}{l}\text { >KIR29693.1 superoxide dismutase [Cu-Zn] [Cryptococcus gattii VGII LA55] } \\
\text { MLAVAVLKGDSPVTGVITFTQEKEGAPVTVSGDIKNLDANAERGFHVHEFG } \\
\text { DNTNGCTSAGPHFNPHGKNHGAPSDSERHVGDLGNVKTDGNGVASVNISD } \\
\text { KSLSLFGPYSIIGRTIVVHAGTDDFGKGGNPESLKTGNAGARAACGVIGISN }\end{array}$ \\
\hline \multirow[t]{3}{*}{4.} & GXM & XP_568628.1 & $\begin{array}{l}\text { >XP_568628.1 O-acetyltransferase [Cryptococcus neoformans var. neoformans } \\
\text { JEC21] } \\
\text { MPNSSKPRSQASAAKLNPLWYTYACATLVAAVVLGNILRWAFLELPDSYH } \\
\text { CSALLNTGKWLDPGTWTNWQPEGCFQLPLSAQSWQKCLASPTVNTHQAL } \\
\text { HSSYYDKRTALFVGDSTVRQLYFAAARKVGKTSKAWELEGEKHTDRSLLV } \\
\text { SDPLGGPSLELEFWWDPYLNSSKTIGLLSGQSSVPSSLLVMGSGLWYLRNPS } \\
\text { SGGLASWGAMIYDTFELVKKNQGSPQTALINPWDNMLLGPGTLPGLLPNQ } \\
\text { PPKFVDHSREVEARSLFSRASSISHRPTDFSISDAIVFLPISTPVREKLSPSRAE } \\
\text { TIFHTDVEAMNADLYARLTHPDPPPVVIPSVLNQLLVDDETEDGLHFSDKIM } \\
\text { NKQAELLLSWRCNDVMRHEGATGTCCKRYDWVTPIQGLILAVLILWAPLG } \\
\text { TFITPRLPPNSPILDYLPATSIAPALSTFGLAMGYLFLADRTHVFQKEQKDYD } \\
\text { AVIFGMITLAAFVAGLLTIKNSGKDLGFLNRDITDEWKGWMQIAILIYHFFG } \\
\text { ASKISGIYNPIRVLVASYLFMTGYGHFFFYYKKADFGFQRVVMVLVLNLL } \\
\text { SVVLPYTMNTDYAFYYFAPLVSWWYLIYYTMAIGSKYNDRPAFLLTKLFT } \\
\text { CAGLVTLFMHFPWLMEDVFKVLNTVFNIQWSAKEWSFRVTLDLFIVWVG } \\
\text { MLCAYGFVKFNEHQISDRPWFPVMRTATLVGSVLGMIWYFWFELHLASKF } \\
\text { VYNEYHAVVCIVPIMSFVFLRNASPVLRSSTSKIFCFIGQCSLETFILQFHGW } \\
\text { LASDTKAILLAVPSTQWRPVNLVISTICFIWLSYRVSGATGEITEWLVGKKK } \\
\text { ALPLPATSANSSTSPGRQATSPTLTSASAMQAVVVGPQDGAKGGIPESIPMM } \\
\text { NQADKDIGGLTPMEDETLERRDSWPTWMASTAASLTGRTVEGYAPLTRRW } \\
\text { KDQTVLSVIQNLGDLMKKHNSVKIAVILLGLWALNWIY }\end{array}$ \\
\hline & & $\begin{array}{l}\text { XP_01205355 } \\
4.1\end{array}$ & $\begin{array}{l}\text { >XP_012053554.1 O-acetyltransferase [Cryptococcus neoformans var. grubii H99] } \\
\text { MPNSSNSRSQATAAKLNPLWYTYACATLVAAVVLGNILRWAFLELPDSYH } \\
\text { CSALLNTGKWLDPGTWTNWPEGCFQLPLSAQSWQRCLASPTVNTHQAL } \\
\text { HSYYDKRTALFVGDSTVRQLYFATARKVGKASKAWESEGEKHTDRSLLVS } \\
\text { DPLGGPSLELEFWWDPYLNSSKTIGLLSGRGLAPSSLLVMGSGLWYLRNPS } \\
\text { SGGLASWGAMIHDTFEFIKKNQGSPQAALINPWDNMLLGSGLTLPGLLPQQ } \\
\text { SPKFVDSSREVEARSLFSRASSASHRPADFSISDAIVYLPISTPVHEKLSSSRA } \\
\text { ETIFHTDVEAMNADLYARLTHPDPPPVVIPSVFNQLLVDDETEDGLHFSDKI } \\
\text { MDKQAELLLSWRCNDVMRHEGATGACCKRYDWVTPIQGLILAVLILWAPL } \\
\text { GTFITPRLPPNSPIHDYLPSPSIAPALSTFGLAVGYLFLADRTHVFQKEQKDY } \\
\text { DAVVFGVITFAAFVAGLLTIKNSGKDLGFLNRDITDEWKGWMQIAILIYHFF } \\
\text { GASKISGIYNPIRVLVASYLFMTGYGHFFFYYKKADFGFQRVVMVLVRLNL } \\
\text { LSVVLPYTMNTDYAFYYFAPLVSWWYLIIYATMAFGSKYNDRPAFLLAKL } \\
\text { FTCAGLVTLFMHFPWLMEDVFKVLNTVFNIQWSAKEWSFRVTLDLFIVWA } \\
\text { GMLCAYGFVKFKEYQISDRPWFPTMHTATLIGSVLGMIWYFWFELHLANK } \\
\text { FVYNEYHAVVCIVPIISFIFLRNASPVLRSSTSKIFCFIGQCSLETFILQFHGWL } \\
\text { ASDTKAVLLAVPSTQWRPVNLVISTICFIWLSYRVSGATGEITEWLVGKKK } \\
\text { ALPLPATSAGPSTSTSRQATSPTLTTASAMQAVVEGPQDGAKGGIPESIPMM } \\
\text { NQADKDIGGLTPMEDETLERRDSWPTWMASTAASLTGRTAEGYAPLTRQ } \\
\text { WKDQTVLSVIQNLGDLMKKHTSVKIAVILFGLWVLNWIY }\end{array}$ \\
\hline & & KIR84277.1 & $\begin{array}{l}\text { >KIR84277.1 O-acetyltransferase [Cryptococcus gattii VGIV IND107] } \\
\text { MPNSSKRRSQATAAKLNPLWYTYACATLLAAVVLGNFLRWAFLELPDSYH } \\
\text { CSALLNTGKWLDPGTWTNWQPEGCFQLPLPAQSWQKCLASPTVNTHQSLH } \\
\text { SSYFDKRTALFVGDSTVRQLYFAAARKVGKTSKAWESEGEKHTDRSLLVN } \\
\text { DPLGGPSLELEFWWDPYLNSSKTVGLLSGHSPVPSSLLVMGSGLWYLRNPS } \\
\text { SGGLASWGAMIYDTFELFKKNQGSPQTALINPWDNMLLGPGVTLPGLLPD } \\
\text { QPPKSVDYSPEVEARSFLSRASSISRRPTDFSISDAIVFLPISTPVPEKLSPSRAE } \\
\text { TILHTDVEAMNADLYARLTHPDPPPVIMPSVFNQLLVDDETEDGLHFSDKI } \\
\text { MNKQAELLLSWRCNDVMRHEGATGTCCKRYDWVTPVQGLILAILVLWAP }\end{array}$ \\
\hline
\end{tabular}




\begin{tabular}{|c|c|c|c|}
\hline $\begin{array}{l}\text { S.n } \\
\text { o. }\end{array}$ & $\begin{array}{l}\text { Virulence } \\
\text { factors }\end{array}$ & $\begin{array}{l}\text { Accession } \\
\text { No. }\end{array}$ & Sequence in FASTA format \\
\hline & & & $\begin{array}{l}\text { LGTLIAPRLRPKSPVHDYLPSPSIAPALSTFGLAMGYLFLADRTHIFQKEQKD } \\
\text { YDSIIFGMITLAAFVAGLLTVRNSGKDLGFLNRDITDEWKGWMQIAILIYHF } \\
\text { FGASKISGIYNPIRVMVASYLFMTGCEYIAFYYKKADFSFQRVIMVLVRLNL } \\
\text { LSVVLPYTMNTDYAFYYFAPLVSWWYLIIYATMAIGSRYNDRPAFLLPKLFI } \\
\text { CAGLVTLFMHFPWLMADVFKVLNTVFNIQWSAKEWSFRVTLDLFIVWAG } \\
\text { MLCAYGFVKFKEHQISDRPWFPVMRTSTLVGSVLGMIWYLWFELHLPSKF } \\
\text { VYNEYHAVVCVVPIMSFVFLRNASPALRSSTSKIFCFIGQCSLETFILQFHGW } \\
\text { LASDTKAILLAVPSTRWRPVNLVISTICFIWLSYRVSGATGEITEWLIGKKKA } \\
\text { LPPPATSTGPSTSSSRQATTPMFTTASAMQAVVEGPQDGAKGGVPESIPLMN } \\
\text { QADKEVGGLTPVEDETLERRDSWPTWMASTAASFTGRTAEGYAPLTRRW } \\
\text { KDQTVLSVIQNLGDLMKKHNSVKIAVILLGLWILNWIY }\end{array}$ \\
\hline & & KGB79193.1 & $\begin{array}{l}\text { >KGB79193.1 O-acetyltransferase [Cryptococcus gattii VGII R265] } \\
\text { MPNSSKPRSQATAAKLNPLWYTYACATLLAAVVLGNFLRWAFLELPDSYH } \\
\text { CSALLNTGKWLDPGTWTNWQPEGCFQLPLPAQSWQRCLALPTVNTHQSLH } \\
\text { SSYFDKRTALFVGDSTVRQLYFAAARKVGKTSKAWESEGEKHTDRSLLVN } \\
\text { DPLGGPSLELEFWWDPYLNSSKTVGLLSGHSPVPSSLLVMGSGLWYLRNPS } \\
\text { SGGLASWGAMIYDTFELIKKNQGSPQTALINPWDNMLLGPGVTLPGLLPDQ } \\
\text { PLKSDDYSLEVEARSFLSRASSISRRPTDFSISDAIVFLPISTPVPEKLSPSRAET } \\
\text { ILHTDVEAMNADLYARLTHPDPPPVIMPSVFNQLLVDDETEDGLHFSDKIM } \\
\text { NKQAELLLSWRCNDVMRHEGATGTCCKRYDWVTPVQGLILAVLVLWAPF } \\
\text { GTLIAPRLPPKSPVHNYLPSTSIAPALSTFGLAMGYLFLADRTHVFQKEQKD } \\
\text { YDAIIFGTITLAAFVAGLLTVRNSGKDLGFLNRDITDEWKGWMQIAILIYHF } \\
\text { FGASKISGIYNPIRVMVASYLFMTGYGHFFFYYKKADFSFQRVIMVLVRLNL } \\
\text { LSVVLPYTMNTDYAFYYFAPLVSWWYLIIYATMAIGSRYNDRPAFLLPKLFI } \\
\text { CAGLVTLFMHFPWLMADVFKVLNTVFNIQWSAKEWSFRVTLDLFIVWAG } \\
\text { MLCAYGFVKFKEHQISDRPWFPVMRTSALVGSVLGMIWYLWFELHLPSKF } \\
\text { VYNEYHAVVCVVPIMSFVFLRNASPALRSSTSKIFCFIGQCSLETFILQFHGW } \\
\text { LASDTKAILLAVPSTRWRPVNLVISTICFIWLSYRVSGATGEITEWLVGKKK } \\
\text { ALPPPATSTGPSTSSSRQATSPTFTTASAMQAVIEGPQDGAKGGVPESIPLMN } \\
\text { QADKEVGGLTPVEDETLERRDSWPTWMASTAASFTGRTAEGYAPLTRRW } \\
\text { KDQTVLSVIQNLGDLMKKHNSVKIAVILLGLWILNWIY }\end{array}$ \\
\hline & & KIR27425.1 & $\begin{array}{l}\text { >KIR27425.1 O-acetyltransferase [Cryptococcus gattii VGII LA55] } \\
\text { MPNSSKPRSQATAAKLNPLWYTYACATLLAAVVLGNFLRWAFLELPDSYH } \\
\text { CSALLNTGKWLDPGTWTNWQPEGCFQLPLPAQSWQRCLALPTVNTHQSLH } \\
\text { SSYFDKRTALFVGDSTVRQLYFAAARKVGKTSKAWESEGEKHTDRSLLVN } \\
\text { DPLGGPSLELEFWWDPYLNSSKTVGLLSGHSPVPSSLLVMGSGLWYLRNPS } \\
\text { SGGLASWGAMIYDTFELIKKNQGSPQTALINPWDNMLLGPGVTLPGLLPDQ } \\
\text { PLKSDDYSPEVEARSFLSRASSISRRPTDFSISDAIVFLPISTPVPEKLSPSRAET } \\
\text { ILHTDVEAMNADLYARLTHPDPPPVIMPSVFNQLLVDDETEDGLHFSDKIM } \\
\text { NKQAELLLSWRCNDVMRHEGATGTCCKRYDWVTPVQGLILAVLVLWAPF } \\
\text { GTLIAPRLPPKSPVHNYLPSTSIAPALSTFGLAMGYLFLADRTHVFQKEQKD } \\
\text { YDAIIFGTITLAAFVAGLLTVRNSGKDLGFLNRDITDEWKGWMQIAILIYHF } \\
\text { FGASKISGIYNPIRVMVASYLFMTGYGHFFFYYKKADFSFQRVIMVLVRLNL } \\
\text { LSVVLPYTMNTDYAFYYFAPLVSWWYLIIYATMAIGSRYNDRPAFLLPKLFI } \\
\text { CAGLVTLFMHFPWLMADVFKVLNTVFNIQWSAKEWSFRVTLDLFIVWAG } \\
\text { MLCAYGFVKFKEHQISDRSWFPVMRTSALVGSVLGMIWYLWFELHLPSKF } \\
\text { VYNEYHAVVCVVPIMSFVFLRNASPALRSSTSKIFCFIGQCSLETFILQFHGW } \\
\text { LASDTKAILLAVPSTRWRPVNLVISTICFIWLSYRVSGATGEITEWLVGKKK } \\
\text { ALPPPATSTGPSTSSSRQATSPTFTTASAMQAVVEGPQDGAKGGVPESIPLM } \\
\text { NQADKEVGGLTPVEDETLERRDSWPTWMASTAASFTGRTAEGYAPLTRR } \\
\text { WKDQTVLSVIQNLGDLMKKHNSVKIAVILLGLWILNWIY }\end{array}$ \\
\hline & & KIR53077.1 & $\begin{array}{l}\text { >KIR53077.1 O-acetyltransferase [Cryptococcus gattii Ru294] } \\
\text { MPNSSKPRSQATAAKLNPLWYTYACATLLAAVVLGNFLRWAFLELPDSYH } \\
\text { CSALLNTGKWLDPGTWTNWQPEGCFQLPLPAQSWQKCLASPTVNTHQSLH } \\
\text { SSYFDKRTALFVGDSTVRQLYFAAARKVGKTSKAWESEGEKHTDRSLLVN } \\
\text { DPLGGPSLELEFWWDPYLNSSKTVGLLSGHSPVPSSLLVMGSGLWYLRNPS } \\
\text { SGGLASWGAMIYDTFELLKKNQGSPQTALIDPWDNMLLGPGVTLPGLLPD } \\
\text { QPPKSVDYSPEVEARSFLSRASSISRRSTDFSISDAIVFLPISTPVPEKLSPSRAE } \\
\text { TIFHTDVEAMNADLYARLTHPDPPPVIMPSVFNQLLVDDETEDGLHFSDKI } \\
\text { MNKQAELLLSWRCNDVMRHEGATGTCCKRYDWVTPVQGLILAILILWAPL } \\
\text { GTLIAPRLPPKSPVHDYLPSPSIAPALSTFGLAMGYLFLADRTHVFQKEQKD } \\
\text { YDAIIFGTITLAAFVAGLLTVRNSGKDLGFLNRDITDEWKGWMQIAILIYHF } \\
\text { FGASKISGIYNPIRVMVASYLFMTGCEYIAFYYKKADFSFQRVIMVLVRLNL } \\
\text { LSVVLPYTMNTDYAFYYFAPLVSWWYLIIYATMAIGSRYNDRPAFLLPKLFI } \\
\text { CAGLVTLFMHFPWLMADVFKVLNTVFNIQWSAKEWSFRVTLDLFIVWAG } \\
\text { MLCAYGFVKFKEHQISDRPWFPVMRTSTLVGSVLGMIWYLWFELHLPSKF } \\
\text { VYNEYHAVVCVVPIMSFVFLRNASPALRSSTSKIFCFIGQCSLETFILQFHGW } \\
\text { LASDTKAILLAVPSTRWRPVNLVISTICFIWLSYRVSGATGEITEWLVGKKK }\end{array}$ \\
\hline
\end{tabular}




\begin{tabular}{|c|c|c|c|}
\hline $\begin{array}{l}\text { S.n } \\
\text { o. }\end{array}$ & $\begin{array}{l}\text { Virulence } \\
\text { factors }\end{array}$ & $\begin{array}{l}\text { Accession } \\
\text { No. }\end{array}$ & Sequence in FASTA format \\
\hline & & & $\begin{array}{l}\text { ALPPPATSTGPSTSSSRQATSPTFTTASAMQAVVEGPQDGAKGGVPESIPLM } \\
\text { NQADKEVGGLTPVEDETLERRDSWPTWMASTAASFTGRTAEGYAPLTRR } \\
\text { WKDQTVLSVIQNLGDLMKKHNSVKIAVILLGLWILNWIY }\end{array}$ \\
\hline \multirow[t]{6}{*}{5.} & \multirow[t]{6}{*}{$\begin{array}{l}\text { CAP } \\
\text { binding } \\
\text { proteins }\end{array}$} & XP_569639.1 & $\begin{array}{l}\text { >XP_569639.1 cap binding protein, putative [Cryptococcus neoformans var. } \\
\text { neoformans JEC21] } \\
\text { MTSTAIPPAAVAANNNTLNSALAAEQISSPASPVDKPVDEKKQLEEGEIEEN } \\
\text { PSEGDSQTKTIFDDASKFNVKHPLFSTWTLYFDSPQSKSLPKTPQTTPAMPQ } \\
\text { GSHGWMADIRKVVSFDSVEEFWGLYNNIIPPSQLPGKANYYLFKNGIIPAW } \\
\text { EDPQNKNGGKWSIQVPKNSESKSSIDRMWLYTMLAAIGETFETASTDSENA } \\
\text { PSPTQSDLITGVIVSPRPAFYRISIWTREASDVNVLDTDAIKARLLNIGKHFKT } \\
\text { SVLGYELEQKLTEGGFQTEL } \\
\text { TFDAHKDSEKKVNKNKFTV }\end{array}$ \\
\hline & & $\begin{array}{l}\text { XP_00319284 } \\
4.1\end{array}$ & $\begin{array}{l}\text { >XP_003192844.1 cap binding protein, putative [Cryptococcus gattii WM276] } \\
\text { MSSTAIPPAAVSANNNALNSALAAEQISSPASPIDKPEDEKKQLEEGEIEENS } \\
\text { SEGDSQAKTIFDDASKFNVKHPLFSTWTLYFDSPQSKSLPKTPQTTPAMPQG } \\
\text { SHGWMADIRKVVSFDSVEEFWGLYNNIIPPSQLPGKANYYLFKNGIIPAWE } \\
\text { DPQNKNGGKWSIQVPKNSESKGSIDRMWLYTMLAAIGETFETPSTDSENAP } \\
\text { SPIQSDLITGVIVSPRPAFYRISIWTREASDVNVPDTDAIKARLLNIGKHFKTS } \\
\text { VLGYELEQKLTEGGFQTEL } \\
\text { TFDAHKDSEKKVNKNKFTV }\end{array}$ \\
\hline & & $\begin{array}{l}\text { XP_01205279 } \\
3.1\end{array}$ & $\begin{array}{l}\text { >XP_012052793.1 translation initiation factor 4E [Cryptococcus neoformans var. } \\
\text { grubii H99] } \\
\text { MSSTTIPPAAVTANSNTLNSALAAEQISSPASPVGKPVDEKKQLEEGEIEENP } \\
\text { SESDSQTKTIFDDASKFNVKHPLFSTWTLYFDSPQSKSLPKTPQTTPAMPQG } \\
\text { SHGWMADIRKVVSFDSVEEFWGLYNNIIPPSQLPGKANYYLFKNGIIPAWE } \\
\text { DPQNKNGGKWSIQVPKNSESKGSIDRMWLYTMLAAIGETFETPSTESETAPS } \\
\text { PTQSDLITGVIVSPRPAFYRISIWTREASDINIPDTDAIKARLLNIGKHFKTSVL } \\
\text { GYELEQKLTEGGFQTEL } \\
\text { TFDAHKDSEKKVNKNKFTV }\end{array}$ \\
\hline & & KIR87178.1 & $\begin{array}{l}\text { >KIR87178.1 translation initiation factor 4E [Cryptococcus gattii VGIV IND107] } \\
\text { MSSTAIPPAAVSANNNALNSALAAEQISSPASPIDKPEDEKKQLEEGEIEENP } \\
\text { SEGDSQTKTIFDDASKFNVKHPLFSTWTLYFDSPQSKSLPKTPQTTPAMPQG } \\
\text { SHGWMADIRKVVSFDSVEEFWGLYNNIIPPSQLPGKANYYLFKNGIIPAWE } \\
\text { DSQNKNGGKWSIQVPKNSESKGSIDRMWLYTMLAAIGETFETPSTDSENAP } \\
\text { SPTQSDLITGVIVSPRPAFYRISIWTREASDVNVPDTDAIKARLLNIGKHFKTS } \\
\text { VLGYELEQKLTEGGFQTEL } \\
\text { TFDAHKDSEKKVNKNKFTV }\end{array}$ \\
\hline & & KGB77518.1 & $\begin{array}{l}\text { >KGB77518.1 translation initiation factor 4E [Cryptococcus gattii VGII R265] } \\
\text { MSSAAIPPAAVSANNNALNSALAAEQISSPASPIDKPEDEKKQLEEGEIEENP } \\
\text { SEGDSQTKTIFDDASKFNVKHPLFSTWTLYFDSPQSKSLPKTPQTTPAMPQG } \\
\text { SHGWMADIRKVVSFDSVEEFWGLYNNIIPPSQLPGKANYYLFKNGIIPAWE } \\
\text { DPQNKNGGKWSIQVPKNSESKGSIDRMWLYTMLAAIGETFETPSTDSENAP } \\
\text { SPTQSDLITGVIVSPRPAFYRISIWTREASDVNVPDTDAIKARLLNIGKHFKTS } \\
\text { VLGYELEQKLTEGGFQTEL } \\
\text { TFDAHKDSEKKVNKNKFTV }\end{array}$ \\
\hline & & KIR48974.1 & $\begin{array}{l}\text { >KIR48974.1 translation initiation factor 4E [Cryptococcus gattii CA1280] } \\
\text { MSSTAIPPAAVSANNNALNSALAAEQISSPASPIDKPEDEKKQLEEGEIEENP } \\
\text { SEGDSQTKTIFDDASKFNVKHPLFSTWTLYFDSPQSKSLPKTPQTTPAMPQG } \\
\text { SHGWMADIRKVVSFDSVEEFWGLYNNIIPPSQLPGKANYYLFKNGIIPAWE } \\
\text { DPQNKNGGKWSIQVPKNSESKGSIDRMWLYTMLAAIGETFETPSTDSENAP } \\
\text { SPTQSDLITGVIVSPRPAFYRISIWTREASDVNVPDTDAIKARLLNIGKHFKTS } \\
\text { VLGYELEQKLTEGGFQTELTFDAHKDSEKKVNKNKFTV }\end{array}$ \\
\hline \multirow[t]{2}{*}{6.} & \multirow[t]{2}{*}{$\begin{array}{l}\text { Phospholi } \\
\text { pase-B }\end{array}$} & $\begin{array}{l}\text { XP_01205299 } \\
6.1\end{array}$ & $\begin{array}{l}\text { >XP_012052996.1 phospholipase B [Cryptococcus neoformans var. grubii H99] } \\
\text { MSIATATFAFSLFATIAFAVPPETPRIELQAERGLGDKSYAPWQVDCPSNVT } \\
\text { WIRNATTGLGSGERAYIEAREKLVQPVIEQMMAARGLETPPRTPNIGVALS } \\
\text { GGGYRAMLTGLGGIMGMMNESTEASESETGGWLDGVSYWAGLSGGSWA } \\
\text { TGTFMSNGGQLPTNLLENLWNIDSNLVFPDDDKLSFYTELYTETNAKSDLG } \\
\text { FPIQITDVWGLAIGSHVLPERYQLSNTPNLTFSSLPSVVSALGNASLPMPIIIA } \\
\text { AEREAGELVIAENATVWEFTPYEFGSWAFGSQYKSPGAFTPIEYLGTSVDD } \\
\text { GSPNGTCWKGFDQLSFVMGTSATLFNGAFLELNGTDSGLLTNLITAFLADL } \\
\text { GEDQADISRIPNTFSNYNSGENPIYNLTYITLVDAGETNQNIPLEPLLVPTRD } \\
\text { VDAIVAFDSSYDTDYIWPNGTALRTTYERAKVLAEHENTRVLMPEVPSMN } \\
\text { GFVNGGYNSRPTFFGCNDTTTPLIIYVPSYPWSFAANTSTYQLSYENDEANE } \\
\text { MLLNGMRSLTLNHSVPTWPTCFACALTDRSFMYTSENRSTTCQKCFDTWC } \\
\text { WAGDDNTTEPATYEPVINSVPPWLVANNLSIGVADAPASNESTAGTASSGA } \\
\text { AKADVSMGMVALAAGLGLML }\end{array}$ \\
\hline & & AAF61964.1 & >AAF61964.1 phospholipase B [Cryptococcus neoformans var. neoformans] \\
\hline
\end{tabular}




\begin{tabular}{|c|c|c|c|}
\hline $\begin{array}{l}\text { S.n } \\
\text { o. }\end{array}$ & $\begin{array}{l}\text { Virulence } \\
\text { factors }\end{array}$ & $\begin{array}{l}\text { Accession } \\
\text { No. }\end{array}$ & Sequence in FASTA format \\
\hline & & & $\begin{array}{l}\text { MSIITTAFALSLLATTAFAVPPETPRIELQAERGLGDQSYAPWQVDCPSNVT } \\
\text { WIRNATTGLGTGERAYIEAREKLVQPAIEQMMAARGLETPPRTPVIGVALA } \\
\text { GGGYRAMLTGLGGIMGMMNESTEASQSETGGWLDGVSYWSGLSGGSWA } \\
\text { TGSFMSNGGQLPTTLLENLWNIDSNLVFPDDGKLSFYTNLYTETNAKSDLG } \\
\text { FPVQITDIWGLAIGSHVLPEPYQLSNTPNLTFSSLPSVVAALGNASLPMPIIVA } \\
\text { AEREAGELVIAENATVWEFTPYEFGSWAFGSQYKSPGAFTPIEYLGTSVDD } \\
\text { GSPNGTCWKGFDQLSFVMGTSATLFNGAFLELNGTDSGLLTNLITAFLADL } \\
\text { GEDQADISRIPNSFSNYNSGENPIYNLTYITLVDAGETNQNIPLEPLLVPTRD } \\
\text { VDAIVAFDSSYDSDYIWPNGTALRTTYERAKILAEHENTRVLMPEVPSMNG } \\
\text { FVNGGYNSRPTFFGCNDTTTPVIIYIPSYPWSFAANTSTYQLSYENNEANEM } \\
\text { LLNGMRSLTLNHSVPTWPTCFACALTDRSFMYTSENRSTTCQECFDTWCW } \\
\text { AGDDNTTEPANYEPVINSVPPWLIANNLSIGMADAPGSNESTAGTASSGAA } \\
\text { KMGVGMGMVALTAGLGLML }\end{array}$ \\
\hline & & KIR59014.1 & $\begin{array}{l}\text { >KIR59014.1 phospholipase B [Cryptococcus } \text { gattii CA1873] } \\
\text { MSVITTTFALSLFAATALAIPPETPRIELHAERGLGDKSYAPWQVDCPTNVT } \\
\text { WIRNATTGLGSGERAYIEAREKLVQPAIEHMMAARGLETPPRTPVIGVALA } \\
\text { GGGYRAMLTGLGGIMSMMNESTEASESETGGWLEGVSYWSGLSGGSWAT } \\
\text { GTFMSNGGQLPTSLLENLWNIDSNLIFPDDDKVSFYAELYIETNAKSDLGFP } \\
\text { TQITDLWGLAIGSHVLPEQYQLSNNPNLTFSSLPSVVASLGNASLPMPIIIA } \\
\text { EREAGELIIAENATVWEFTPYEFGSWAFGSQYKSPGAFTPIEYLGTSVNDGS } \\
\text { PNGTCWKGFDQLSFVMGTSATLFNGAFLELGTDSGLLTSLITAFLAELGE } \\
\text { DQVDISRIPNTFSNYNSGENPIYNLTYITLVDAGETNQNVPLEPLLIPARAVD } \\
\text { AIVAFDASYDTNYIWPNGTALRTTYERARVLAEHENTRVLMPEVPSMNGF } \\
\text { VNGGYNSRPTFFGCNDTTTPLIIYVPSYPWSFAANTSTYQLSYETDEANQML } \\
\text { LNGMRSLTLNHSVPTWPTCFACALTDRSFMYTSENRSTTCQECFDTWCWA } \\
\text { GDDNTTQPAEYEPVINSVPPWLIANNLSLGVADAPASNESTPGTASSGAAKI } \\
\text { GVSVGMVALAAGLGLMF }\end{array}$ \\
\hline & & KIR38674.1 & $\begin{array}{l}\text { >KIR38674.1 phospholipase B [Cryptococcus gattii VGII Ram5] } \\
\text { MSVITTTFALSLFAVTALAIPPETPRIELQAERGLGDKSYAPWQVDCPTNVT } \\
\text { WIRNATSGLGSGERAYIEAREKLVQPAIEDMMAARGLETPPRTPVIGVALA } \\
\text { GGGYRAMLTGLGGIMSMMNESTEASESETGGWLEGVSYWSGLSGGSWAT } \\
\text { GTFMSNGGQLPTSLLENLWNIDSNLIFPDDDKVSFYTELYTETNAKSDLGFP } \\
\text { TQITDLWGLAIGSHVLPEQYQLSNNPNLTFSSLPSVVAALGNASLPMPIIIAA } \\
\text { EREAGELIIAENATVWEFTPYEFGSWAFGSQYKSPGAFTPIEYLGTSVNDGS } \\
\text { PNGTCWKGFDQLSFVMGTSATLFNGAFLELNGTDSGLLTSLITAFLAELGE } \\
\text { DQADISRIPNTFSNYNSGENPIYNLTYITLVDAGETNQNVPLEPLLVPARVVD } \\
\text { AIVAFDASYDTDYIWPNGTALRTTYERARVLAEHENTRVLMPEVPSMNGF } \\
\text { VNGGYNSRPTFFGCNDTTTPLIIYVPSYPWSFAANTSTYQLSYETDEANQILL } \\
\text { NGMRSLTLNHSVPTWPTCFACALTDRSFMYTSENRSTTCQECFDTWCWAG } \\
\text { DDNTTQPAKYEPVINSVPPWLIANNLSLGVANAPASNESTPGTASSDAAKIG } \\
\text { VSVGIVALAAGLGLMF }\end{array}$ \\
\hline & & KIR84691.1 & $\begin{array}{l}\text { >KIR84691.1 phospholipase B [Cryptococcus } \text { gattii VGIV IND107] } \\
\text { MSVIATTFALSIFAATALAIPPETPPIELQAERGLGDKSYAPWQVDCPTNVT } \\
\text { WIRNATTGLGSGERAYIEAREKLVQPAIEHMMAARGLKTPPRTPVIGVALA } \\
\text { GGGYRAMLTGLGGIMSMMNESTEASESETGGWLEGVSYWSGLSGGSWAT } \\
\text { GTFMSNGGQLPTSLLENLWNIDSNLIFPDDDKVSFYTELYTETNAKSDLGFP } \\
\text { TQITDLWGLAIGSHVLPEQYQLSNNPNLTFSSLPSVVAALGNASLPMPIIIAA } \\
\text { EREAGELIIAENATVWEFTPYEFGSWAFGSQYKSPGAFTPIEYLGTSVNDGS } \\
\text { PNGTCWKGFDQLSFVMGTSATLFNGAFLELGTDSGLLTSLITAFLAELGE } \\
\text { DQVDISRIPNTFSNYNSGENPIYNLTYITLVDAGETNQNVPLEPLLVPARAVD } \\
\text { AIVAFDASYDTDYIWPNGTALRTTYERARVLAEHENTRVLMPEVPSMNGF } \\
\text { VNGGYNSRPTFFGCNDTTTPLIIYVPSYPWSFAANTSTYQLSYETDEANQILL } \\
\text { NGMRSLTLNHSVPTWPTCFACALTDRSFMYTSENRSTICQECFDTWCWAG } \\
\text { DDNTTKPAEYEPVINSVPPWLIANNLSLGVADAPASNGSIPGTASSGAAKIG } \\
\text { VSVGMVALAAGLGLMF }\end{array}$ \\
\hline & & KIR52753.1 & $\begin{array}{l}\text { >KIR52753.1 phospholipase B [Cryptococcus } \text { gattii Ru294] } \\
\text { MSVITTTFALSLFAATALAIPPETPRIELQAERGLGDKSYAPWQVNCPTNVT } \\
\text { WIRNATTGLGSGERAYIEAREKLVQPAIEHMMAARGLETPPRTPVIGVALA } \\
\text { GGGYRAMLTGLGGIMSMMNESTEASESETGGWLEGVSYWSGLSGGSWAT } \\
\text { GTFMSNGGQLPTSLLENLWNIDSNLIFPDDDKVSFYTELYTETNAKSDLGFP } \\
\text { TQITDLWGLAIGSHVLPQQYQLSNNPNLTFSSLPSVVAALGNASLPMPIIIAA } \\
\text { EREAGELIIAENATVWEFTPYEFGSWAFGSQYKSPGAFTPIEYLGTSVNDGS } \\
\text { PNGTCWKGFDQLSFVMGTSATLFNGAFLELNGTDSGLLTSL } \\
\text { ITAFLAELGEDQADISRIPNTFSNYNSGENPIYNLTYITLVDAGETNQNVPLE } \\
\text { PLLIPARAVDAIVAFDASYDTDYIWPNGTALRTTYERARVLAEHENTRVLM } \\
\text { PEVPSMNGFVNGGYNSRPTFFGCNDTTTPLIIYVPSYPWSFAANTSTYQLSY } \\
\text { ETDEANQILLNGMRSLTLNHSVPTWPTCFACALTDRSFMYTSENRSTICQEC }\end{array}$ \\
\hline
\end{tabular}




\begin{tabular}{|c|c|c|c|}
\hline \multirow[t]{2}{*}{$\begin{array}{l}\text { S.n } \\
\text { o. } \\
\end{array}$} & $\begin{array}{l}\text { Virulence } \\
\text { factors }\end{array}$ & $\begin{array}{l}\text { Accession } \\
\text { No. }\end{array}$ & Sequence in FASTA format \\
\hline & & & $\begin{array}{l}\text { FDTWCWAGDDNTTQPAEYEPVINSVPPWLIANNLSLGVADAPASNESTPGT } \\
\text { ASSGAAKIGVSVGMVALAAGLGLMF }\end{array}$ \\
\hline \multirow[t]{5}{*}{7.} & \multirow[t]{5}{*}{ GalXM } & XP_568018.1 & $\begin{array}{l}\text { >XP_568018.1 cryptococcal xylosyltransferase } 1 \text { [Cryptococcus neoformans var. } \\
\text { neoformans JEC21] } \\
\text { MPLNLPFSSPLKLPLPRRFIILILSASILILFLHTFAPSTLPPVLTPNLPHHEPDA } \\
\text { SYFSPSKWLPPILNPNAPTRPLEFDEDGQCLFLSPFDALSAAEKARARVLSLD } \\
\text { EISPGIVRADAPPAEGTDADPDFDDEFSELSNATRKMPAGLTHPILGLLRDG } \\
\text { EAKWNSMVTMQSQTLEQAVDVYMDRWGRPPKGFDEWWHFAKANNVL } \\
\text { LPDEYDPIMNSLLPFYALPIDTLKERLVEAEKIPETFTLIVHDGKVELKWND } \\
\text { DYSRDTWWASRPRADSQINLMEPFIKHIGTFRATFTIHDQPSILLDHERQEEL } \\
\text { LTAARHGKISTHPNELDRAEQNWRKACPPDSPLNKGEEELAPDSFISSHLA } \\
\text { AMDICQHPSYMENHGMLLEEKNSDSHPKPHTKLYPILVPSKTALNGDIPVTP } \\
\text { IGKDGRRDDIGHDPEWNRKSGKLYWRGLATGLQHNKKAGAKWRQSHRER } \\
\text { LHFLANDKSDTYTEVLSPVGSSGEAELARMPLRELGQYMDVKLAGGNW } \\
\text { QCDWGDGTCEEMEKEIDFAPKDSSERSNDFKYVFDTDGNAWSSRFPRLMA } \\
\text { SNNVVIKSTVFPEWNTNSLPEWYAYVPSKMDYSDLFSIMTFFRTPSGRGA } \\
\text { HDEVARRIALNGQCWVERTWRREDLQAYMFRLYLEYARLTSPDRDNGKM } \\
\text { DYVSTQKKASKEADDVPAAADIETPVIDQ }\end{array}$ \\
\hline & & KIR46264.1 & $\begin{array}{l}\text { >KIR46264.1 beta-1,2-xylosyltransferase } 1 \text { [Cryptococcus gattii CA1280] } \\
\text { MPLNLPFSSALKLPLPRRFIILILSASILILFLHTFAPSTLPPVLTPNLQHHEPDA } \\
\text { SYFSPSKWLPPILNPNAPTRPLEFDEEGQCLFLSPFDALSAAEKARAQVLSLN } \\
\text { EISPGIVRAEAPPAEGTDADPDFDDEFSELSNATRKMPTGLTHPILGLLRDGE } \\
\text { AKWNSMLARQSQTLEEAVNVYIERWGRKPPKGFDEWWHFAKSNNVLLPD } \\
\text { EYDAIMNSLLPFYALPIETLKERLAEAEKIPETFTLIVHDGKVEQWNDDYS } \\
\text { RDTWWASRPRADSQINLMEPFIKHIGTFRATFTIHDQPSVLLDYERQKELLT } \\
\text { AARQGKISTHPNEIDRAEQNWKKACAPDSPLNKGEEELEASDSFISSHLAA } \\
\text { MDICQHPSYLENHGMLLEEKNSDTHPKPHTKLYPILVPSTALNGDIPVTPI } \\
\text { GKDGRRDDVGHDPEWSRKSGKLYWRGLATGLQHNKKAGAKWRQSHRER } \\
\text { LHFLANDKSDAYTEVLSPVGSSGEAELAQMPKELGQYYMDVKLAGGNW } \\
\text { QCDWGDGTCEEMEKEIDFAPKDSSERSNDFKYVFDTDGNAWSSRFPRLMA } \\
\text { SNNVVIKSTVFPEWNTNSLPEWYAYVPSKMDYSDLFSIMTFFRGTPSGRGA } \\
\text { HDEVARRIALNGQCWVERTWRREDLQAYMFRLYLEYARLTSPDRDNGKM } \\
\text { DYVPTPKKISNVAHGVHVAADVEPPVDQ }\end{array}$ \\
\hline & & KIR75920.1 & $\begin{array}{l}\text { >KIR75920.1 beta-1,2-xylosyltransferase } 1 \text { [Cryptococcus gattii VGII CA1014] } \\
\text { MPLNLPFSSALKLPLPRRFIILILSASILILFLHTFAPSTLPPVLTPNLQHHEPDA } \\
\text { SYFSPSKWLPPILNPNAPTRPLEFDEEGQCLFLSPFDALSAAEKARAQVLSLN } \\
\text { EISPGIVRAEAPPAEGTDADPDFDDEFSELSNATRKMPPGLTHPILGLLRDGE } \\
\text { AKWNSMLARQSQTLEEAVNVYIERWGRKPPKGFDEWWHFAKANNVLLPD } \\
\text { EYDAIMNSLLPFYALPIETLKERLTEAEKIPETFTLIVHDGKVELQWNDDYS } \\
\text { RDTWWASRPRADSQINLMEPFIKHIGTFRATFTIHDQPSVLLDYERQKELLT } \\
\text { AARQGKISTHPNEIDRAEQNWKKACAPDSPLNKGEEELEASDSFISSHLAA } \\
\text { MDICQHPSYLENHGMLLEEKNSDTHPKPHTKLYPILVPSKTALNGDIPVTPI } \\
\text { GKDGRRDDVGHDPEWSRKSGKLYWRGLATGLQHNKKAGAKWRQSHRER } \\
\text { LHFLANDKSDAYTEVLSPVGSSGEAELAQMPLKELACRWKLAM }\end{array}$ \\
\hline & & KIR85290.1 & $\begin{array}{l}\text { >KIR85290.1 beta-1,2-xylosyltransferase } 1 \text { [Cryptococcus gattii VGIV IND107] } \\
\text { MPLNLPFSSALKLPLPRRFIILILSASILILFLHTFAPSTLPPVLTPNLQHHEPDA } \\
\text { SYFSPSKWLPPILNPNAPTRPLEFDEEGQCLFLSPFDALSAAEKARAQVLSLN } \\
\text { EISPGIVRAEAPPAEGTDADPDFDDEFSELSNATRKMPTGLTHPILGLLRDGE } \\
\text { AKWNSMLARQSQTLEEAVNVYIERWGRKPPKGFDEWWHFAKANNVLLPD } \\
\text { EYDAIMNSLLPFYALPIETLKERLTEAEKIPETFTLIVHDGKVELWNDDYS } \\
\text { RDTWWASRPRADSQINLMEPFIKHIGTFRATFTIHDQPSVLLDYERQKELLT } \\
\text { AARQGKISTHRNEIDRAEQNWKKACAPDSPLNKGEEELEASDSFISSHLAA } \\
\text { MDICQHPSYLENHGMLLEEKNADTHPKPHTKLYPILVPSKTALNGDIPVTPI } \\
\text { GKDGRRDDVGHDPEWSRKSGKLYWRGLATGLQHNKKAGAKWRQSHRER } \\
\text { LHFLANDKSDAYTEVLSPVGSSGEAELAQMPKELGQYYMDVKLAGGNW } \\
\text { QCDWGDGTCEEMEKEIDFAAKDSSERSNDFKYVFDTDGNAWSSRFPRLMA } \\
\text { SNNVVIKSTVFPEWNTNSLPEWYAYVPSKMDYSDLFSIMTFFRGTPSGRGA } \\
\text { HDEVARRIALNGQCWVERTWRREDLQAYMFRLYLEYARLTSPDRDNGKM } \\
\text { DYVPTPKKISNVAHGVPVAADVEPPVDQ }\end{array}$ \\
\hline & & $\begin{array}{l}\text { XP_01204874 } \\
0.1\end{array}$ & $\begin{array}{l}\text { >XP_012048740.1 beta-1,2-xylosyltransferase } 1 \text { [Cryptococcus neoformans var. } \\
\text { grubii H99] } \\
\text { MPLNLPFSSPLLPLPRRFIILILSASILILFLHTFAPSTLPPVFTPSLPHHEPDAS } \\
\text { YFSPSKWLPPILNPNAPTRPLEFDENGQCLFLSPFDALSAAEKARAQVLSLD } \\
\text { EVSPGIVRADAPPAEGTDADPDFDDEFSELSNATRKMPAGLTHPILGLLRDG } \\
\text { EAKWNSMLARQSQTLEQAVDVYIDRWGRKPPKGFDEWWHFAKANNVLL } \\
\text { PDEYDPIMNSLLPFYALPIDTLEERLIEAEKIPETFTLIVHDGKVELKWNDDY } \\
\text { SRDTWWASRPRADSQINLMEPFIKHIGTFRATFTIHDQPSILLDHERHEELLT } \\
\text { AARHGKVSTHPNELDRAEQNWKKACPPDSPLNKGEVELEAPDSFISSHLAA }\end{array}$ \\
\hline
\end{tabular}




\begin{tabular}{|c|c|c|c|}
\hline \multirow[t]{3}{*}{$\begin{array}{l}\text { S.n } \\
\text { o. } \\
\end{array}$} & $\begin{array}{l}\text { Virulence } \\
\text { factors }\end{array}$ & $\begin{array}{l}\text { Accession } \\
\text { No. }\end{array}$ & Sequence in FASTA format \\
\hline & & & $\begin{array}{l}\text { MDICQHPSYMENHGMLLEEKNSDTHPKPHTKLYPILVPSKTALNGDIPVTPI } \\
\text { GKDGRRDDIGHDPEWSRKSGKLYWRGLATGLQHNKKAGAKWRQSHRER } \\
\text { LHFLANDKSDTYTEVLSPVGSTGEAELAQMPLRELGQYYMDVKLAGGNW } \\
\text { QCDWGDGTCDEMEKEIDFAPKDSSERSNDFKYVFDTDGNAWSSRFPRLMA } \\
\text { SNNVVIKSTVFPEWNTNSLPEWYAYVPSKMDYSDLFSIMTFFRGTPSGRGA } \\
\text { HDEVARRIALNGQCWVERTWRREDLQAYMFRLYLEYARLTSPDRDNGKM } \\
\text { DYIPSQERP }\end{array}$ \\
\hline & & KIR54007.1 & $\begin{array}{l}\text { >KIR54007.1 beta-1,2-xylosyltransferase } 1 \text { [Cryptococcus gattii Ru294] } \\
\text { MPLNLPFSSALKLPLPRRFIILILSASILILFLHTFAPSTLPPVLTPNLQHHEPDA } \\
\text { SYFSPSKWLPPILNPNAPTRPLEFDEEGQCLFLSPFDALSAAEKARAQVLSLN } \\
\text { EISPGIVRAEAPPAEGTDADPDFDDEFSELSNATRKMPTGLTHPILGLLRDGE } \\
\text { AKWNSMLARQSQTLEEAVNVYIERWGRKPPKGFDEWWHFAKSNNVLLPD } \\
\text { EYDAIMNSLLPFYALPIETLKARLAEAEKIPETFTLIVHDGKVELQWNDDYS } \\
\text { RDTWWASRPRADSQINLMEPFIKHIGTFRATFTIHDQPSVLLDYERQKELLT } \\
\text { AARQGKISTHPNEIDRAEQNWKKACAPDSPLNKGEEELEASDSFISSHLAA } \\
\text { MDICQHPSYLENHGMLLEEKNSDTHPKPHTKLYPILVPSKTALNGDIPVTPI } \\
\text { GKDGRRDDVGHDPEWSRKSGKLYWRGLATGLQHNKKAGAKWRQSHRER } \\
\text { LHFLANDKSDAYTEVLSPVGSSGEAELAQMPLKELGQYYMDVKLAGGNW } \\
\text { QCDWGDGSCEEMEKEIDFAPKDSSERSNDFKYVFDTDGNAWSSRFPRLMA } \\
\text { SNNVVIKSTVFPEWNTNSLPEWYAYVPSKMDYSDLFSIMTFFRGTPSGRGA } \\
\text { HDEVARRIALNGQCWVERTWRREDLQAYMFRLYLEYARMTSPDRDNGK } \\
\text { MDYVPTPKKASNVAHGVPVAADVEPPVNQ }\end{array}$ \\
\hline \multirow[t]{5}{*}{8.} & \multirow[t]{5}{*}{ MP } & XP_567104.1 & $\begin{array}{l}\text { >XP_567104.1 } 88 \mathrm{kDa} \text { immunoreactive mannoprotein } \mathrm{MP88,} \text { putative } \\
\text { [Cryptococcus neoformans var. neoformans JEC21] } \\
\text { MISKVALGAAAALMAGVANVNAQVTATGTMGPTNPSEPTLGTAINQTSYA } \\
\text { RLLSLNAIDDFCLFAPPEPDSVIGDTEAEEVAWCVQPRNNARVIPDGVLTAV } \\
\text { HFVKTPLYWQIQGFGDFTHLNIQDGDEGGELDPHGATGLGNPVGGNVTTN } \\
\text { ATGSDVSYEEWMNYMAYDQFCLRICISENSTYSAANECQHTLDEMGCSWV } \\
\text { MPGDYTNNSFTECDGDSAYPPGWYILANGSTSTFQQRYTTYTNGDGSLG } \\
\text { TWTQGETVTPQTAYSTPATSNCKTYTSVGNGIASLALSNAGSVNSTAAATN } \\
\text { SSSGGASAAATGSSSSGSTAGSSAGSSAGSGSGSAAAGSTAAASSSGDSSSS } \\
\text { TSAAMSNGINYGTAMAGVISVVALVAGAGSFLL }\end{array}$ \\
\hline & & CAC78985.1 & $\begin{array}{l}\text { >CAC78985.1 macrofage activating glycoprotein [Cryptococcus neoformans var. } \\
\text { neoformans] } \\
\text { MGPTNPPEPTLGTPINQTSYARLLSLNAIDDFCLFAPPEPDSVIGDTEAEEVA } \\
\text { WCVQPRNNARVIPDGVLTAVHFVKTPLYWQIQGFGDFTHLNIQDGDEGGE } \\
\text { LDPHGATGLGNPVGGNVTTNATGSDVSYEEWMNYMAYDQFCLRICISENS } \\
\text { TYSAANECQHTLDEMGCSWVMPGDYTNNSFTECDGDSAYPPGWYILANG } \\
\text { STSTFQQRYTGTYTNGDGSLGTWTQGETVTPQTAYSTPATSNCKTYTSVGN } \\
\text { GIASLALSNAGSVNSTAAATNSSSGGASAAATGSSSSGSTAGSSAGSSAGSG } \\
\text { SGSAAAGSTAAASSSGDSSSSTSAAMSNGINYGTAMAGVISVVALVAGAGS } \\
\text { FLL }\end{array}$ \\
\hline & & $\begin{array}{l}\text { XP_01204695 } \\
3.1\end{array}$ & $\begin{array}{l}\text { >XP_012046953.1 immunoreactive mannoprotein MP88 [Cryptococcus neoformans } \\
\text { var. grubii H99] } \\
\text { MISKVAVGAAAALMAGVANVNAQVTATGTMGPTNPPEPTLGTPINQTSYA } \\
\text { RLLSLNAIDDFCLFAPPVPNSVIGETEAEEVAWCVQPRNNARVIPDGVLTAV } \\
\text { HFVKTPLYWQIQGFGDFTHLNIQSGDEGGELDPHGATGLGNPVGGNVTTN } \\
\text { ATGSDVSYEEWMNYMAADQFCLRICISENSTYSAANECQHTLDEMGCSWV } \\
\text { MPGDYTADSFTECDGDSAYPPGWYILGNGSTSTFQQRYGTFTGADGSLGT } \\
\text { WTQGETVTPQSAYSTPASSNCKTYTSVGNGIASLALSNAGSVNSTASATNSS } \\
\text { SGGSSAAATRSSSSGSSAGSGSGSAVAGSTAAASSSGESSSSTSAAMSSFNGI } \\
\text { SYGTAMAVVALVAGAGSFLL }\end{array}$ \\
\hline & & KGB75317.1 & $\begin{array}{l}\text { >KGB75317.1 immunoreactive mannoprotein MP88 [Cryptococcus gattii VGII } \\
\text { R265] } \\
\text { MGCAPDGKWQSSPHVSFHHPSNVAIGAAAALMAGVANVNAQVTATGTM } \\
\text { GPTNPPAATLGTAINQTSYARLLSLNAIDDFCLFAPPVPNSVIGETEAEEVA } \\
\text { WCVQPRNDARVIPDGVLTAVHFVKTPLYWQIQGFGDFTHLNIQSGDEGGEL } \\
\text { DPHGATGLGNPVGGNVTTNATGSDVSYEEWMNYMAFDQFCLRICISENDT } \\
\text { YSAANECQHTLDEMGCSWVMPGDYTNNSFTECDGDSAYPPGWYPEANGS } \\
\text { TSTFQQRYGTFTNADGSLGTWTQGETVTPQSAYSIPATSNCKTYTSVGNGI } \\
\text { SSLALSNAGSVNSTAASTGSSSGSSPAAATGSSSSGGSGASGSANAGSTAAA } \\
\text { SASGSSSKSAAMSSFSGVNYGSAVAGAISVVALVAGAGSFLL }\end{array}$ \\
\hline & & KIR51504.1 & $\begin{array}{l}\text { >KIR51504.1 immunoreactive mannoprotein MP88 [Cryptococcus gattii Ru294] } \\
\text { MISKVAIGAAAALMAGVANVNAQVTATGTMGPTNPPAATLGTAINQTSYA } \\
\text { RLLSLNAIDDFCLFAPPVPNSVIGETEAEEVAWCVQPRNDARVIPDGVLTAV } \\
\text { HFVKTPLYWQIQGFGDFTHLNIQSGDEGGELDPHGATGLGNPVGGNVTTNS } \\
\text { TGSDVSYEEWMNYMAFDQFCLRICISENDTYSAANECQHTLDEMGCSWV } \\
\text { MPGDYTNNSFTECDGDSAYPPGWYPEANGSTSTFQQRYTGTFTNADGSLG }\end{array}$ \\
\hline
\end{tabular}




\begin{tabular}{|c|c|c|c|}
\hline $\begin{array}{l}\text { S.n } \\
\text { o. }\end{array}$ & $\begin{array}{l}\text { Virulence } \\
\text { factors }\end{array}$ & $\begin{array}{l}\text { Accession } \\
\text { No. }\end{array}$ & Sequence in FASTA format \\
\hline & & & $\begin{array}{l}\text { TWTQGQTVTPQSAYSVPATSNCKTYTSVGNGIASLALSNAGSVNSTGASSG } \\
\text { SSSSNSPAAATGSSSSSGGSGASGSANAGSTAAASASGSSSNSGAMSSFSGV } \\
\text { NYGSAMAGAISVVALVAGAGSFLL }\end{array}$ \\
\hline & & KIR83819.1 & $\begin{array}{l}\text { >KIR83819.1 immunoreactive mannoprotein MP88 [Cryptococcus gattii VGIV } \\
\text { IND107] } \\
\text { MISKVAIGAAAALMAGVANVNAQVTATGTMGPTNPPAATLGTAINQTSYA } \\
\text { RLLSLNAIDDFCLFAPPVPNSVIGETEAEEVAWCVQPRNDARVIPDGVLTAV } \\
\text { HFVKTPLYWQIQGFGDFTHLNIQNGDEGGELDPHGATGLGNPVGGNVTTN } \\
\text { STGSDVSYEEWMNYMSFNQFCLRICISENDTYSAANECQHTLDEMGCSWV } \\
\text { MPGDYTNNSFTECDGDSAYPPGWYPEANGSTSTFQQRYTGTFTNADGSLG } \\
\text { TWTQGQTVTPQSAYSVPATSNCKTYTSVGNGISSLALSNAGSVNSTAASSG } \\
\text { SSSGNSPAAATGSSSSGGSGASGSANAGSTAAASASGSSTKSGAMSSFSGVN } \\
\text { YGSAMAGAISVVALVAGAGSFLL }\end{array}$ \\
\hline
\end{tabular}

The pathogenic potential of virulence factors of Cryptococcus was evaluated in-silico using orthologs. We retrieved 48amino acid sequences of different virulence factors of Cryptococcus species. In 2019, Elhassan et al. retrieved 38 amino acid sequences of heat shock $70 \mathrm{KDa}$ protein of $C$. n.grubii from the NCBI protein database and used them for in-silico epitopes prediction and vaccine development [36]. In another study, Sati et al. retrieved 28 amino acid sequences of pyruvate kinase protein of $C$. albicans from NCBI and were subjected to multiple sequence alignment and epitope prediction [37]. In another study, Schneider et al. (2015) retrieved sequences of four zinc transporter genes of C. gattii R265 for phylogenetic analysis [38].

\subsection{Maximum likelihood test and hierarchical clustering of virulence proteins of} Cryptococcus species.

The shape of a gamma distribution is a statistical numerical parameter showing variation. Its higher value indicates the low variation of substitution rates among sites. Amino acid sequences of laccase, CAP binding protein, and mannoprotein (MP) of different Cryptococcus species with gamma distribution values 97.967, 97.898, and 97.759, respectively, were found to be highly conserved (Table 2). In contrast, GalXM, GXM, and SOD's low gamma distribution values represent moderate and high rates of variation, respectively. Amongst all virulence factors, superoxide dismutase (SOD) with the lowest gamma distribution value (0.907) was considered as highly adaptable (Table 2) and susceptible to selection pressure.

Table 2.Gamma distribution parameter for virulence factors of Cryptococcus species.

\begin{tabular}{l|l|l} 
S.No. & Virulence Factors & Gamma distribution parameter \\
\hline 1 & SOD & 0.907 \\
\hline 2 & GalXM & 1.088 \\
\hline 3 & GXM & 1.312 \\
\hline 4 & Phospholipase B & 8.300 \\
\hline 5 & Urease & 37.894 \\
\hline 6 & MP & 97.759 \\
\hline 7 & CAP binding protein & 97.898 \\
\hline 8 & Laccase & 97.967
\end{tabular}

Hierarchical clustering grouped the virulence factors of C. n. neoformans, C. n. grubii, and $C$. gattii into 8 clusters and 48 cluster orders. It also demonstrated the formation of homogenous groups of variables and phylogenetic evolutionary relationship among different virulence factors of Cryptococcus species (Figure 1). Laccase, CAP binding protein, and MP 
showed maximum similarity as compared to other virulence factors. SOD proteins, however, were observed to be distantly located in the dendrogram.

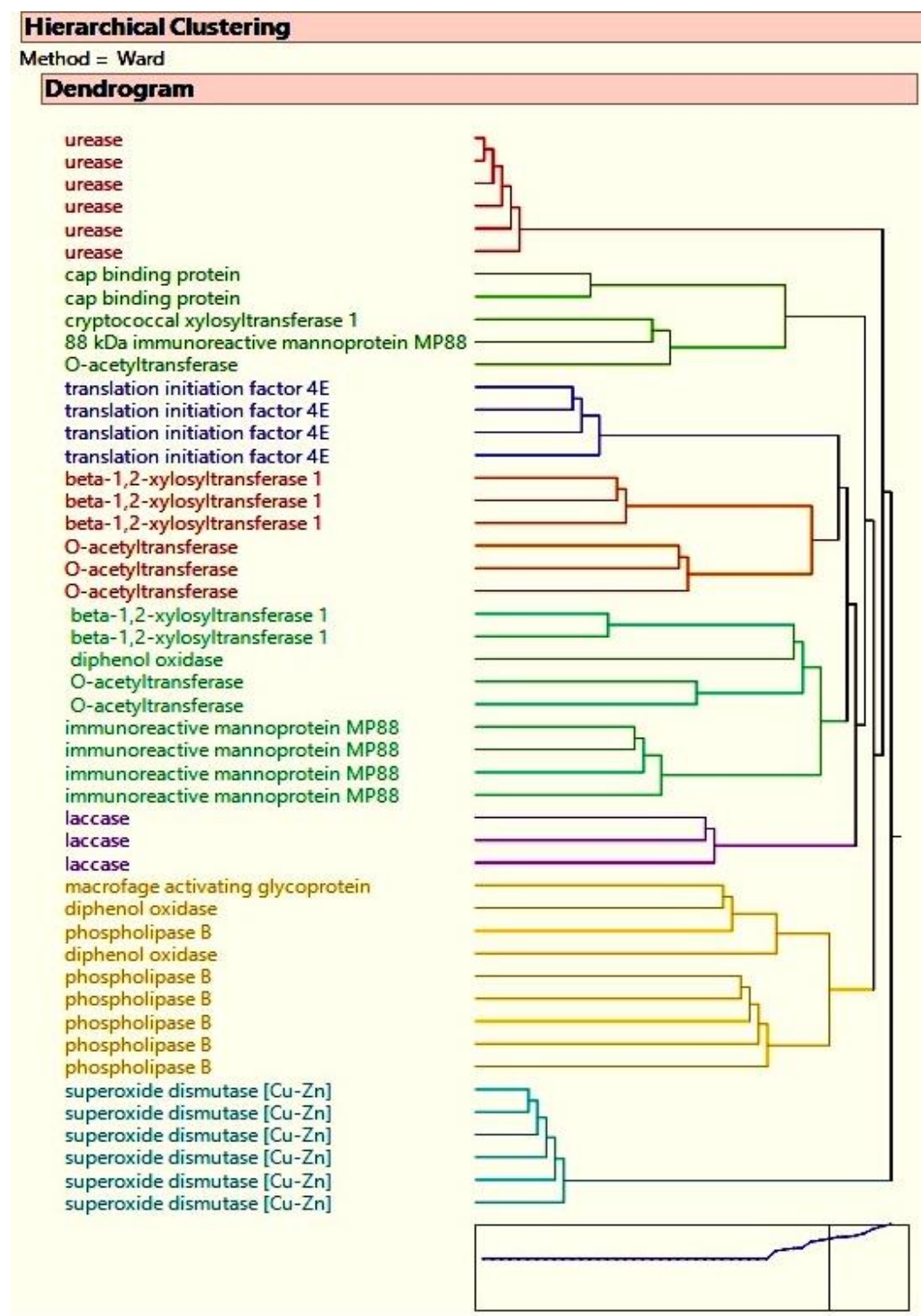

Figure 1. Phylogenetic relationship and hierarchical clustering of virulence factors of Cryptococcus species

Phylogenetic analysis in the present study recognized a core set of virulence proteins in the pathogenic species of Cryptococcus. The orthologs were used to detect the evolution rate, which was further clustered hierarchically to assess the relationship and variation amongst the virulence proteins of Cryptococcus species. All virulence proteins except SOD were grouped separately in one cluster.

$\mathrm{Cu}, \mathrm{Zn}$ superoxide dismutase encoded by the SOD1 gene is crucial for the pathogenesis of Cryptococcus[16]. The low estimated value of the gamma distribution parameter symbolizes a high rate of variation in substitution. Unlike its highly conserved mammalian counterparts [39], $\mathrm{Cu}, \mathrm{Zn}$ SODof Cryptococcus was highly variable and can be governed by natural selection pressure. The resultant adaptability might enhance its importance in cryptococcal pathogenesis.

\subsection{B-cell linear epitope prediction in virulence proteins of Cryptococcus species.}

The consensuses epitopes were obtained from three web servers ABCpred, BCPred, and BcePred (Table 1). The virulence factors of $C . n$. grubii were found to have more B-cell 
epitopes than those of $C$. $n$. neoformans and $C$. gattii. The maximum number of predicted epitopes was observed in the urease of $C$. n. grubii.

Table 1. Consensuses of linear epitopes of virulence factors in Cryptococcus sps. predicted by online web servers.

\begin{tabular}{|c|c|c|c|c|}
\hline $\begin{array}{ll}\text { Species } & \text { of } \\
\text { Cryptococcus } & \\
\end{array}$ & Virulence factors & $\begin{array}{l}\begin{array}{l}\text { No. of } \\
\text { epitopes }\end{array} \\
\end{array}$ & $\begin{array}{l}\text { Start } \\
\text { Position }\end{array}$ & Predicted epitope residues \\
\hline \multirow{32}{*}{ C.n. neoformans } & \multirow{4}{*}{ Cap Binding Protein } & 1 & 89 & SKSLPKTPQT \\
\hline & & 2 & 41 & KKQLEEGEIEENP \\
\hline & & 3 & 200 & STDSENAPSPT \\
\hline & & 4 & 174 & SESKSS \\
\hline & \multirow{6}{*}{ GalXM } & 1 & 73 & APTRPLEFD \\
\hline & & 2 & 344 & SPLNKGEEELE \\
\hline & & 3 & 673 & KASKEAD \\
\hline & & 4 & 635 & TWRREDL \\
\hline & & 5 & 523 & TCEEMEKEID \\
\hline & & 6 & 258 & WNDDYSRD \\
\hline & \multirow{2}{*}{ GXM } & 1 & 327 & HPDPPPVVIPSVL \\
\hline & & 2 & 831 & SSTSPGRQAT \\
\hline & \multirow{4}{*}{ Laccase } & 1 & 230 & TNCTATNSS \\
\hline & & 2 & 570 & NTDPNSFGPARRSPSPSIQ \\
\hline & & 3 & 176 & QRGRDYDEDR \\
\hline & & 4 & 48 & PTTREYT \\
\hline & \multirow{4}{*}{ MP } & 1 & 317 & ASSSGDSSSST \\
\hline & & 2 & 277 & SGGASAAATGSSSSG \\
\hline & & 3 & 206 & TFQQRYTGT \\
\hline & & 4 & 96 & QDGDEGG \\
\hline & \multirow{5}{*}{ Phospholipase-B } & 1 & 600 & PGSNESTAGTAS \\
\hline & & 2 & 484 & VIIYIP \\
\hline & & 3 & 569 & NTTEPAN \\
\hline & & 4 & 127 & ASQSE \\
\hline & & 5 & 371 & SFSNYNSG \\
\hline & \multirow{3}{*}{ SOD } & 1 & 53 & DNTNGCT \\
\hline & & 2 & 128 & GKGGNEESLK \\
\hline & & 3 & 78 & AERHV \\
\hline & \multirow{4}{*}{ Urease } & 1 & 253 & HKDQEKVEEGPT \\
\hline & & 2 & 427 & PADGSNATTCT \\
\hline & & 3 & 656 & GDEPTRDNNRVKR \\
\hline & & 4 & 460 & TGKGNDSG \\
\hline \multirow{19}{*}{ C.n. grubii } & \multirow{4}{*}{ Cap Binding Protein } & 1 & 89 & SKSLPKTPQT \\
\hline & & 2 & 41 & KKQLEEGEIEENPSESDS \\
\hline & & 3 & 199 & PSTESETAPSPT \\
\hline & & 4 & 160 & NKNGG \\
\hline & \multirow{5}{*}{ GalXM } & 1 & 73 & APTRPLEF \\
\hline & & 2 & 634 & TWRREDL \\
\hline & & 3 & 523 & TCDEMEKEI \\
\hline & & 4 & 331 & RAEQNWK \\
\hline & & 5 & 419 & GKDGRRDD \\
\hline & \multirow{5}{*}{ GXM } & 1 & 835 & SRQAT \\
\hline & & 2 & 856 & PQDGA \\
\hline & & 3 & 245 & PGLLPQQSP \\
\hline & & 4 & 134 & WESEGE \\
\hline & & 5 & 889 & LERRDSW \\
\hline & \multirow{4}{*}{ Laccase } & 1 & 219 & EGYKGSP \\
\hline & & 2 & 320 & NTSEGKE \\
\hline & & 3 & 183 & NEPLQRGRDYDEDR \\
\hline & & 4 & 362 & STEEPQT \\
\hline & MP & 1 & 338 & ASSSGESSS \\
\hline
\end{tabular}




\begin{tabular}{|c|c|c|c|c|}
\hline $\begin{array}{ll}\text { Species } & \text { of } \\
\text { Cryptococcus } & \end{array}$ & Virulence factors & $\begin{array}{l}\text { No. of } \\
\text { epitopes }\end{array}$ & $\begin{array}{l}\text { Start } \\
\text { Position }\end{array}$ & Predicted epitope residues \\
\hline & & 2 & 299 & ASATNSSSGGSS \\
\hline & & 3 & 270 & ASSNCKT \\
\hline & \multirow{6}{*}{ Phospholipase B } & 1 & 600 & PASNESTAGTAS \\
\hline & & 2 & 90 & ETPPRTP \\
\hline & & 3 & 569 & NTTEPAT \\
\hline & & 4 & 485 & IIYVPS \\
\hline & & 5 & 123 & STEASES \\
\hline & & 6 & 504 & LSYENDEA \\
\hline & \multirow{3}{*}{ SOD } & 1 & 69 & KKHHG \\
\hline & & 2 & 75 & PTDSER \\
\hline & & 3 & 126 & DLGKGGNEESLK \\
\hline & \multirow{7}{*}{ Urease } & 1 & 253 & HKEQEKIE \\
\hline & & 2 & 427 & PADGSNATTCT \\
\hline & & 3 & 656 & GDEPTRDNNRVKR \\
\hline & & 4 & 318 & GGQASGRHD \\
\hline & & 5 & 460 & TGKGSDSG \\
\hline & & 6 & 204 & EPGEKKT \\
\hline & & 7 & 784 & SIGKKDMK \\
\hline \multirow{33}{*}{ C. gattii } & \multirow{5}{*}{ Cap Binding Protein } & 1 & 35 & DKPEDEKKQLEEGEIEE \\
\hline & & 2 & 89 & SKSLPKTPQT \\
\hline & & 3 & 197 & ETPSTDSENA \\
\hline & & 4 & 160 & NKNGG \\
\hline & & 5 & 171 & PKNSESKGS \\
\hline & & 1 & 73 & APTRPLEFDE \\
\hline & & 2 & 345 & PLNKGEEELEA \\
\hline & & 3 & 658 & PDRDNGK \\
\hline & GalXM & 4 & 419 & GKDGRRDDVGH \\
\hline & & 5 & 634 & RTWRREDL \\
\hline & & 6 & 270 & SRPRADS \\
\hline & & 1 & 373 & HEGAT \\
\hline & GXM & 2 & 133 & KAWESEGEKHTD \\
\hline & & 1 & 176 & RGRDYDEDR \\
\hline & Laccase & 2 & 309 & NTSEGKE \\
\hline & & 3 & 48 & PTTREYT \\
\hline & & 1 & 313 & ATGSSSSSGGSGASGS \\
\hline & MP & 2 & 296 & NSTGASSGSSSS \\
\hline & & 3 & 149 & VTTNSTGS \\
\hline & & 1 & 600 & PASNESTPGTAS \\
\hline & & 2 & 568 & NTTQPAEY \\
\hline & Phospholipase B & 3 & 485 & NTTQPAEY \\
\hline & & 4 & 127 & ASESE \\
\hline & & 5 & 372 & FSNYNSG \\
\hline & & 1 & 20 & TQEKE \\
\hline & SOD & 2 & 125 & DFGKGG \\
\hline & & 3 & 88 & KTDGNG \\
\hline & & 1 & 253 & HKEQEKVE \\
\hline & & 2 & 427 & PADGSNATTC \\
\hline & Urens & 3 & 656 & GDEPTRDNNRVKR \\
\hline & Urease & 4 & 460 & TGKGNDSGT \\
\hline & & 5 & 204 & EPGEKKT \\
\hline & & 6 & 582 & LMVCHHLD \\
\hline
\end{tabular}

Acquaintance about virulence is also essential to identify potent antifungal targets. In 2018, Khalil and co-workers predicted 11 conserved antigenic B-cell epitopes in MP88 (mannoprotein) of $C$. neoformans and designed a peptide vaccine against it using in-silico simulations [40]. However, the current study utilized sequences of virulence proteins of $C . n$. 
neoformans, C. n. grubii, and C. gattii for linear B cell-binding epitope prediction. Silva et al. (2019) highlighted new methodologies using bioinformatic tools and servers that have led to the development of new vaccines, like peptide-based vaccines [41].

As humans lack urease, a nickel-containing enzyme [42], therefore, the B-cell epitopes of $C . n$. grubii urease viz HKEQEKIE, PADGSNATTCT, GDEPTRDNNRVKR, GGQASGRHD, TGKGSDSG, EPGEKKT, and SIGKKDMK predicted in this study could be useful in designing and developing of the epitope-based vaccine against cryptococcal infections.

\section{Conclusions}

Induction of adaptation and production of true virulence factors are two important processes in cryptococcal virulence. The study reveals the phylogenetic relationship among orthologs of virulence proteins of $C$. n. neoformans, C. $n$. grubii, and C. gattii. Superoxide dismutase (SOD) proteins of Cryptococcus exhibited independent evolvement and high variability. Thus could contribute significantly to the pathogenicity of Cryptococcus. This study further performed B-cell epitope prediction using a bioinformatics approach and reports urease as a target for epitope-based anti-cryptococcal drugs. However, the results should be corroborated by experimental studies.

\section{Funding}

This research received no external funding.

\section{Acknowledgments}

The authors are thankful to the Principal, MMV, for the infrastructure facilities.

\section{Conflicts of Interest}

The authors declare no conflict of interest.

\section{References}

1. Ajello, L. The medical mycological iceberg, p. In Proc: Xth Intern. Symposium Mycoses, PAHO Scient. Publ., Washington DC, 1970.

2. Munivenkataswamy, R.; Gopi, A.; Shaik Mohammed Usman, J. Human immunodefeciency virus associated cryptococcal meningitis at a tertiary care centre: diagnostic tools and antifungal susceptibility testing. Journal of clinical and diagnostic research: JCDR 2013, 7, 1623, https://doi.org/10.7860/JCDR/2013/6147.3271.

3. Kronstad, J.; Saikia, S.; Nielson, E.D.; Kretschmer, M.; Jung, W.; Hu, G.; Geddes, J.M.H.; Griffiths, E.J.; Choi, J.; Cadieux, B.; Caza, M.; Attarian, R. Adaptation ofCryptococcus neoformans to Mammalian Hosts: Integrated Regulation of Metabolism and Virulence. Eukaryot. Cell 2012, 11, 109, https://doi.org/10.1128/EC.05273-11.

4. Cherniak, R.; Sundstrom, J.B. Polysaccharide antigens of the capsule of Cryptococcus neoformans. Infect. Immun. 1994, 62, 1507-1512, https://doi.org/10.1128/IAI.62.5.1507-1512.1994.

5. Casadevall, A.; Coelho, C.; Cordero, R.J.B.; Dragotakes, Q.; Jung, E.; Vij, R.; Wear, M.P. The capsule of Cryptococcus neoformans. Virulence 2019, 10, 822-831,https://doi.org/10.1080/21505594.2018.1431087.

6. de Oliveira, H.C.; Trevijano-Contador, N.; Garcia-Rodas, R. Cryptococcal Pathogenicity and Morphogenesis. Current Fungal Infection Reports 2019, 13, 67-76,https://doi.org/10.1007/s12281-019-00340-y.

7. Kozel, T.R.; Levitz, S.M.; Dromer, F.; Gates, M.A.; Thorkildson, P.; Janbon, G. Antigenic and Biological Characteristics of Mutant Strains of Cryptococcus neoformansLacking Capsular O Acetylation or Xylosyl Side Chains. Infect. Immun. 2003, 71, 2868, https://doi.org/10.1128/IAI.71.5.2868-2875.2003. 
8. Probert, M.; Zhou, X.; Goodall, M.; Johnston, S.A.; Bielska, E.; Ballou, E.R.; May, R.C. A Glucuronoxylomannan Epitope Exhibits Serotype-Specific Accessibility and Redistributes towards the Capsule Surface during Titanization of the Fungal PathogenCryptococcus neoformans. Infect. Immun. 2019, 87, e00731-00718, https://dx.doi.org/10.1128\%2FIAI.00731-18.

9. De Jesus, M.; Moraes Nicola, A.; Chow, S.-K.; Lee, I.R.; Nong, S.; Specht, C.A.; Levitz, S.M.; Casadevall, A. Glucuronoxylomannan, galactoxylomannan, and mannoprotein occupy spatially separate and discrete regions in the capsule of Cryptococcus neoformans. Virulence 2010, 1, 500508,https://doi.org/10.4161/viru.1.6.13451.

10. Toplis, B.; Bosch, C.; Schwartz, I.S.; Kenyon, C.; Boekhout, T.; Perfect, J.R.; Botha, A. The virulence factor urease and its unexplored role in the metabolism of Cryptococcus neoformans. FEMS Yeast Res. 2020, 20,https://doi.org/10.1093/femsyr/foaa031.

11. Olszewski, M.A.; Noverr, M.C.; Chen, G.-H.; Toews, G.B.; Cox, G.M.; Perfect, J.R.; Huffnagle, G.B. Urease expression by Cryptococcus neoformans promotes microvascular sequestration, thereby enhancing central nervous system invasion. The American journal of pathology 2004, 164, 1761-1771, https://doi.org/10.1016/S0002-9440(10)63734-0.

12. Almeida, F.; Wolf, J.M.; Casadevall, A. Virulence-Associated Enzymes of Cryptococcus neoformans. Eukaryot. Cell 2015, 14, 1173,https://doi.org/10.1128/EC.00103-15.

13. Zaragoza, O. Basic principles of the virulence of Cryptococcus. Virulence 2019, 10, 490-501, https://doi.org/10.1080/21505594.2019.1614383.

14. Williamson, P.R. Laccase and melanin in the pathogenesis of Cryptococcus neoformans. Front. Biosci. 1997, 2, e99-107, https://doi.org/10.2741/a231.

15. Park, Y.-D.; Chen, S.H.; Camacho, E.; Casadevall, A.; Williamson, P.R. Role of the ESCRT Pathway in Laccase Trafficking and Virulence ofCryptococcus neoformans. Infect. Immun. 2020, 88, e0095400919,https://doi.org/10.1128/IAI.00954-19.

16. Narasipura, S.D.; Ault, J.G.; Behr, M.J.; Chaturvedi, V.; Chaturvedi, S. Characterization of Cu,Zn superoxide dismutase (SOD1) gene knock-out mutant of Cryptococcus neoformans var. gattii: role in biology and virulence. Mol. Microbiol. 2003, 47, 1681-1694,https://doi.org/10.1046/j.1365-2958.2003.03393.x.

17. Smith, A.D.; Garcia-Santamarina, S.; Ralle, M.; Loiselle, D.R.; Haystead, T.A.; Thiele, D.J. Transcription factor-driven alternative localization of Cryptococcus neoformans superoxide dismutase. J. Biol. Chem. 2021, 100391, https://doi.org/10.1016/j.jbc.2021.100391.

18. Cox, G.M.; Harrison, T.S.; McDade, H.C.; Taborda, C.P.; Heinrich, G.; Casadevall, A.; Perfect, J.R. Superoxide Dismutase Influences the Virulence of \&lt;em\&gt;Cryptococcus neoformans\&lt;/em\&gt; by Affecting Growth within Macrophages. Infect. Immun. 2003, 71, 173,https://doi.org/10.1128/IAI.71.1.173180.2003.

19. Narasipura, S.D.; Chaturvedi, V.; Chaturvedi, S. Characterization of Cryptococcus neoformans variety gattii SOD2 reveals distinct roles of the two superoxide dismutases in fungal biology and virulence. Mol. Microbiol. 2005, 55, 1782-1800,https://doi.org/10.1111/j.1365-2958.2005.04503.x.

20. Santangelo, R.; Zoellner, H.; Sorrell, T.; Wilson, C.; Donald, C.; Djordjevic, J.; Shounan, Y.; Wright, L. Role of Extracellular Phospholipases and Mononuclear Phagocytes in Dissemination of Cryptococcosis in a Murine Model. Infect. Immun. 2004, 72, 2229,https://doi.org/10.1128/IAI.72.4.2229-2239.2004.

21. Buchanan, K.L.; Murphy, J.W. What makes Cryptococcus neoformans a pathogen? Emerging Infect. Dis. 1998, 4, 71-83,https://doi.org/10.3201/eid0401.980109.

22. Soria-Guerra, R.E.; Nieto-Gomez, R.; Govea-Alonso, D.O.; Rosales-Mendoza, S. An overview of bioinformatics tools for epitope prediction: Implications on vaccine development. J. Biomed. Inf. 2015, 53, 405-414,https://doi.org/10.1016/j.jbi.2014.11.003.

23. Potocnakova, L.; Bhide, M.; Pulzova, L.B. An Introduction to B-Cell Epitope Mapping and In Silico Epitope Prediction. Journal of Immunology Research 2016, 2016, 6760830, https://doi.org/10.1155/2016/6760830.

24. Larsen, J.E.P.; Lund, O.; Nielsen, M. Improved method for predicting linear B-cell epitopes. Immunome Res. 2006, 2, 1-7, https://doi.org/10.1186/1745-7580-2-2.

25. Sun, P.; Ju, H.; Liu, Z.; Ning, Q.; Zhang, J.; Zhao, X.; Huang, Y.; Ma, Z.; Li, Y. Bioinformatics Resources and Tools for Conformational B-Cell Epitope Prediction. Comput. Math. Methods Med. 2013, 2013, 943636, https://doi.org/10.1155/2013/943636.

26. Raoufi, E.; Hemmati, M.; Eftekhari, S.; Khaksaran, K.; Mahmodi, Z.; Farajollahi, M.M.; Mohsenzadegan, M. Epitope Prediction by Novel Immunoinformatics Approach: A State-of-the-art Review. Int. J. Pept. Res. Ther. 2020, 26, 1155-1163,https://doi.org/10.1007/s10989-019-09918-z. 
27. Casadevall, A.; Coelho, C.; Alanio, A. Mechanisms of Cryptococcus neoformans-Mediated Host Damage. Front. Immunol. 2018, 9, 855, https://doi.org/10.3389/fimmu.2018.00855.

28. Hole, C.R.; Lam, W.C.; Upadhya, R.; Lodge, J.K. Cryptococcus neoformansChitin Synthase 3 Plays a Critical Role in Dampening Host Inflammatory Responses. mBio 2020, 11, e0337303319,https://doi.org/10.1128/mBio.03373-19.

29. Han, L.-T.; Wu, L.; Liu, T.-B. A Predicted Mannoprotein Cmp1 Regulates Fungal Virulence in Cryptococcus neoformans. Pathogens 2020, 9,https://doi.org/10.3390/pathogens9110881.

30. Edgar, R.C. MUSCLE: a multiple sequence alignment method with reduced time and space complexity. BMC Bioinformatics 2004, 5, 113, https://doi.org/10.1186/1471-2105-5-113.

31. Guindon, S.; Lethiec, F.; Duroux, P.; Gascuel, O. PHYML Online-a web server for fast maximum likelihood-based phylogenetic inference. Nucleic Acids Res. 2005, 33, W557W559,https://doi.org/10.1093/nar/gki352.

32. Galanis, K.A.; Nastou, K.C.; Papandreou, N.C.; Petichakis, G.N.; Iconomidou, V.A. Linear B-cell epitope prediction: a performance review of currently available methods. bioRxiv 2019,https://doi.org/10.1101/833418.

33. Coelho, C.; Bocca, A.L.; Casadevall, A. Chapter One - The Tools for Virulence of Cryptococcus neoformans. In Adv. Appl. Microbiol., Sariaslani, S., Gadd, G.M., Eds. Academic Press: 2014; 87, 1-41, https://doi.org/10.1016/b978-0-12-800261-2.00001-3.

34. Ma, H.; May, R.C. Chapter 5 Virulence in Cryptococcus Species. In Adv. Appl. Microbiol., Academic Press: 2009; 67, 131-190, https://doi.org/10.1016/S0065-2164(08)01005-8.

35. Maliehe, M.; Ntoi, M.A.; Lahiri, S.; Folorunso, O.S.; Ogundeji, A.O.; Pohl, C.H.; Sebolai, O.M. Environmental Factors That Contribute to the Maintenance of Cryptococcus neoformans Pathogenesis. Microorganisms 2020, 8,https://dx.doi.org/10.3390\%2Fmicroorganisms8020180.

36. Elhassan, R.M.; Alsony, N.M.; Othman, K.M.; Izz-Aldin, D.T.; Alhaj, T.A.; Ali, A.A.; Abashir, L.A.; Ahmed, O.H.; Hassan, M.A. Computational vaccinology approach: Designing an efficient multi-epitope peptide vaccine against \&lt;em\&gt;Cryptococcus neoformans var. grubii's\&lt;/em\&gt; heat shock 70KDa protein. bioRxiv 2019,https://doi.org/10.1101/534008.

37. Sati, A.O.M.; Abdelmoneim Hamza, A.H.; Dawoud, E.D.K.; Abbas, T.A.A.; Abdelrahman, F.A.B.; Saad, K.A.H.; Abduallah, R.B.A.; Mustafa, M.M.E.S.; Sidahmed, T.a.; Elhassan, R.M.; Hassan, M.A. Multiepitope Peptide Vaccine Prediction for Candida albicans targeting Pyruvate Kinase Protein; an Immunoinformatics Approach. bioRxiv 2019,https://doi.org/10.1101/758920.

38. Schneider, R.d.O.; Diehl, C.; dos Santos, F.M.; Piffer, A.C.; Garcia, A.W.A.; Kulmann, M.I.R.; Schrank, A.; Kmetzsch, L.; Vainstein, M.H.; Staats, C.C. Effects of zinc transporters on Cryptococcus gattii virulence. Sci. Rep. 2015, 5, 10104,https://doi.org/10.1038/srep10104.

39. Robinett, N.G.; Peterson, R.L.; Culotta, V.C. Eukaryotic copper-only superoxide dismutases (SODs): A new class of SOD enzymes and SOD-like protein domains. J. Biol. Chem. 2018, 293, 4636-4643, https://doi.org/10.1074/jbc.TM117.000182.

40. Khalil, I.; Omer, I.; Farh, I.Z.A.; Mohamed, H.A.; Elsharif, H.A.; Mohamed, A.A.H.; Awad-Elkareem, M.A.E.; Salih, M.A. Design of an epitope-based peptide vaccine againstCryptococcus neoformans. BioRxiv2018, 1, 434779, https://doi.org/10.1101/434779.

41. B. R. Da Silva, L.; P. Taborda, C.; D. Nosanchuk, J. Advances in Fungal Peptide Vaccines. Journal of Fungi 2020, 6,https://doi.org/10.3390/jof6030119.

42. Rutherford, J.C. The Emerging Role of Urease as a General Microbial Virulence Factor. PLoS Path. 2014, 10, e1004062, https://doi.org/10.1371/journal.ppat.1004062. 